Linköping Studies in Science and Technology

Dissertation No. 2071

\title{
Contributions to linear discriminant analysis with applications to growth curves
}

Edward Kanuti Ngailo

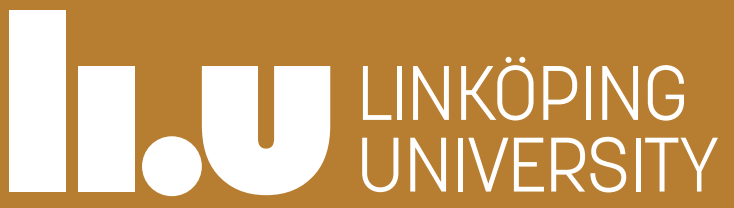


Linköping Studies in Science and Technology.

Dissertations. No. 2071

\section{Contributions to linear discriminant analysis with applications to growth curves}

\section{Edward Kanuti Ngailo}

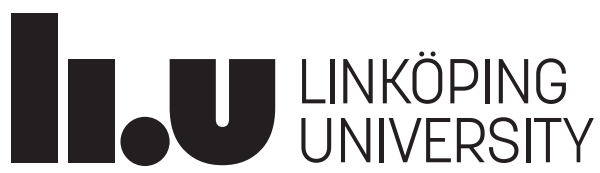

Department of Mathematics, Division of Mathematical Statistics Linköping University, SE-581 83 Linköping, Sweden Linköping 2020 
Linköping Studies in Science and Technology.

Dissertations. No. 2071

Contributions to linear discriminant analysis with applications to growth curves

Edward Kanuti Ngailo

Division of Mathematical Statistics

Department of Mathematics

Linköping University

SE-581 83 Linköping

Sweden

edward.ngailo@liu.se

https://Iiu.se/en/organisation/liu/mai/ms

ISBN 978-91-7929-856-2 ISSN $\quad$ 0345-7524

Copyright @ 2020 Edward Kanuti Ngailo

Printed by LiU-Tryck, Linköping, Sweden 2020 

To my family 


\section{Abstract}

Edward Kanuti Ngailo (2020). Contributions to linear discriminant analysis with applications to growth curves.

Doctoral dissertation. ISBN: 978-91-7929-856-2. ISSN: 0345-7524.

This thesis concerns contributions to linear discriminant analysis with applications to growth curves.

Firstly, we present the linear discriminant function coefficients in a stochastic representation using random variables from the standard univariate distributions. We apply the characterized distribution in the classification function to approximate the classification error rate. The results are then extended to large dimension asymptotics under assumption that the dimension $p$ of the parameter space increases together with the sample size $n$ to infinity such that the ratio $\frac{p}{n}$ converges to a positive constant $c \in(0,1)$.

Secondly, the thesis treats repeated measures data which correspond to multiple measurements that are taken on the same subject at different time points. We develop a linear classification function to classify an individual into one out of two populations on the basis of the repeated measures data that when the means follow a growth curve structure. The growth curve structure we first consider assumes that all treatments (groups) follows the same growth profile. However, this is not necessarily true in general and the problem is extended to linear classification where the means follow an extended growth curve structure, i.e., the treatments under the experimental design follow different growth profiles.

At last, a function of the inverse Wishart matrix and a normal distribution finds its application in portfolio theory where the vector of optimal portfolio weights is proportional to the product of the inverse sample covariance matrix and a sample mean vector. Analytical expressions for higher order moments and non-central moments of the portfolio weights are derived when the returns are assumed to be independently multivariate normally distributed. Moreover, the expressions for the mean, variance, skewness and kurtosis of specific estimated weights are obtained. The results are complemented using a Monte Carlo simulation study, where data from the multivariate normal and $t$-distributions are discussed. 



\section{Sammanfattning}

Den här avhandlingen studerar diskriminantanalys, klassificering av tillväxtkurvor och portföljteori.

Diskriminantanalys och klassificering är flerdimensionella tekniker som används för att separera olika mängder av objekt och för att tilldela nya objekt till redan definierade grupper (så kallade klasser). En klassisk metod är att använda Fishers linjära diskriminantfunktion och när alla parametrar är kända så kan man enkelt beräkna sannolikheterna för felklassificering. Tyvärr är så sällan fallet, utan parametrarna måste skattas från data, och då blir Fishers linjära diskriminantfunktion en funktion av en Wishartmatris och multivariat normalfördelade vektorer. I den här avhandlingen studerar vi hur man kan approximativt beräkna sannolikheten för felklassificering under antagande att dimensionen på parameterrummet ökar tillsammans med antalet observationer genom att använda en särskild stokastisk representation av diskriminantfunktionen.

Upprepade mätningar över tiden på samma individ eller objekt går att modellera med så kallade tillväxtkurvor. Vid klassificering av tillväxtkurvor, eller rättare sagt av upprepade mätningar för en ny individ, bör man ta tillvara på både den spatiala- och temporala informationen som finns hos dessa observationer. Vi vidareutvecklar Fishers linjära diskriminantfunktion att passa för upprepade mätningar och beräknar asymptotiska sannolikheter för felklassificering.

Till sist kan man notera att snarlika funktioner av Wishartmatriser och multivariat normalfördelade vektorer dyker upp när man vill beräkna de optimala vikterna i portföljteori. Genom en stokastisk representation studerar vi egenskaperna hos portföljvikterna och gör dessutom en simuleringsstudie för att förstå vad som händer när antagandet om normalfördelning inte är uppfyllt. 



\section{Acknowledgments}

First and foremost, I would like to thank my supervisor Dr. Martin Singull for his continuous support in every aspect of my studies. Thank you for all the interesting discussions about mathematics and beyond, in particular for your advice. I am also very grateful to my co-supervisor, Professor Dietrich von Rosen, for the fruitful discussions, for working with me and for great support. I am thankful to Professor Taras Bodnar for working with me, for his guidance during the first years, and for stimulating to work at the Stockholm University.

I am also greatful to Dr. Stepan Mazur, Dr. Nestor Parolya and Dr. Farrukh Javed for the pleasant collaborations.

I wish to thank the Department of Mathematics for creating a good environment. Special thanks go to Bengt-Ove Turesson, Theresa Lagali Hansen and Meaza Abebe for their excellent support. I am grateful to Monica, Karin, Mathilda, Theresia, for their great support.

I appreciate the financial support of the Swedish International Development Agency (Sida) through Tanzania-Sweden bilateral programme.

I would like to extend my thanks to Dr. Sylvester Rugeihyamu for his support since I started my PhD studies. Also I am thankful to Dr. Shaban Mbare for his support.

I would also like to thank Stefane, Stanislas, Denise, Emelyne, Beatrice and other colleagues at the Division of Mathematical Statistics for their friendship and support.

Finally, I wish to thank my family for their endless love, support and encouragement.

Linköping, May 4, 2020

Edward Kanuti Ngailo 



\section{Contents}

1 Introduction 1

1.1 Background ....................... 1

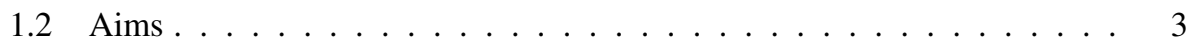

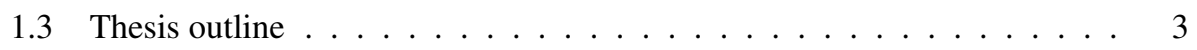

1.3.1 Outline of Part I . . . . . . . . . . . . . . 3

1.3.2 Outline of Part II . . . . . . . . . . . . . . . . 4

1.4 Author's contributions .................. 5

$\begin{array}{lll}\text { I } & \text { Theoretical background } & 7\end{array}$

2 Multivariate normal distribution and Wishart distribution 9

2.1 Normal distribution . . . . . . . . . . . . . . . . . . 9

2.2 Wishart distribution ................... 11

2.2.1 Wishart and non-central Wishart distribution . . . . . . . . 11

2.2.2 Moments of the inverted Wishart distribution . . . . . . . . . . 12

2.3 The Growth Curve model . . . . . . . . . . . . . . . . . . . . 13

3 Discriminant analysis $\quad 21$

3.1 Introduction . . . . . . . . . . . . . . . . . . . 21

3.2 Stochastic representation of the discriminant function . . . . . . . . . . 22

3.3 Classification rule . . . . . . . . . . . . . . . . . . . 24

3.4 Large dimension asymptotic approach . . . . . . . . . . . . . 26 
3.5 Classification of growth curves . . . . . . . . . . . . . . . . 28

3.6 Applications to Portfolio theory . . . . . . . . . . . . . . 33

4 Concluding Remarks 39

4.1 Summary of contributions . . . . . . . . . . . . . . 39

4.2 Future research . . . . . . . . . . . . . . . . . . . 40

Bibliography 41

$\begin{array}{ll}\text { II Papers } & 47\end{array}$

A Discriminant analysis in small and large dimensions 49

1 Introduction . . . . . . . . . . . . . . . . . . . . 52

2 Finite-sample properties of the discriminant function . . . . . . . . . . . 54

2.1 Stochastic representation for the discriminant function coefficients 55

2.2 Test for the population discriminant function coefficients . . . . 58

2.3 Classification analysis . . . . . . . . . . . . 60

3 Discriminant analysis under large-dimensional asymptotics . . . . . . . 65

3.1 Classification analysis in high dimension . . . . . . . . . . 68

3.2 Finite-sample performance . . . . . . . . . . 70

References . . . . . . . . . . . . . . . . . . . . . 74

B Approximation of misclassification probabilities in linear discriminant analysis with repeated measurements

1 Introduction . . . . . . . . . . . . . . . . . . . 82

2 Classification into one of two growth curves . . . . . . . . . . . . 84

$2.1 \quad$ Estimators of the parameters $\mathbf{B}$ and $\mathbf{\Sigma} \ldots \ldots \ldots 85$

3 Approximation of probabilities of misclassification . . . . . . . . . 86

$3.1 \quad$ Approximation of misclassification errors, known $\Sigma \ldots$. . . 86

3.2 Approximation of misclassification errors, unknown $\boldsymbol{\Sigma} \ldots \ldots 88$

4 Simulation study . . . . . . . . . . . . . . . . . . . . . 94

5 Summary ............................ 96

References . . . . . . . . . . . . . . . . . . 97

C Linear discriminant analysis via the Growth Curve model and restrictions $\begin{array}{ll}\text { on the mean space } & 101\end{array}$

1 Introduction . . . . . . . . . . . . . . . . . . . . . . . . . . 104

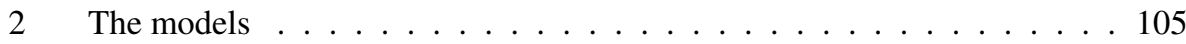

3 Estimators of parameters in three models . . . . . . . . . . . . . . 109 
4 Linear discriminant functions . . . . . . . . . . . . . . . . . . 113

5 Approximation of misclassification errors . . . . . . . . . . . . . . . . . 114

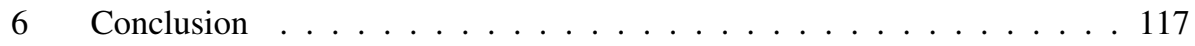

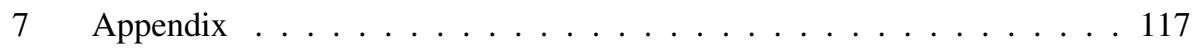

References . . . . . . . . . . . . . . . . . 118

D Higher order moments of the estimated tangency portfolio weights 121

1 Introduction . . . . . . . . . . . . . . . . . . . . . . . . 124

2 Main Results . . . . . . . . . . . . . . . . . . 126

3 Application Implications of Main Results . . . . . . . . . . . . . . . 130

4 Auxiliary Results . . . . . . . . . . . . . . . . . . . . . 132

5 Simulation Studies and Application _ . . . . . . . . . . . . 135

5.1 Simulation Studies . . . . . . . . . . . . . . . 135

$5.2 \quad$ Application .................... . . 137

6 Conclusions . . . . . . . . . . . . . . . . . . 137

References . . . . . . . . . . . . . . . . . . . . 142 


\section{Introduction}

M ultivariate analysis is an important direction of statistics which analyses several variables simultaneously. In practice, multiple variables data sets appear commonly and we usually concern ourselves with several features of the observations. Many multivariate statistical methods discussed in the literature rely on the assumption that the observation vectors are independent and normally distributed. The main reason for this is that sets of multivariate observations are often at least approximately normally distributed. Moreover, the normal distributions are mathematically convenient to handle and many useful explicit results can be found. Discriminant analysis is one of the multivariate statistical methods. This thesis concerns contributions to linear discriminant analysis with applications to growth gurves.

\subsection{Background}

In the late 1920's and in the 1930's multivariate statistics was developing and attracted researchers. The first one to deal with what today is known as discriminant analysis was Fisher (1936). It can be noted that the early development of discriminant analysis such as in Pearson (1916) did not include correlations between the different variables in the feature vector, and considered only the differences between groups based on sample moments. Later, Fisher published four papers on discriminant analysis including Fisher (1938) which he reviewed his work of 1936, and related it to the contributions by Hotelling (1931) and Mahalanobis (1936). In particular, Fisher introduced the concept of linear discriminant function to distinguish between two sets of observations assumed to have distributions with the same covariance matrix. Follow-up papers written on dis- 
criminant analysis and its use include Anderson (1951), John (1961), Okamoto (1963), Gupta (1968), Lachenbruch (1968), McLachlan (1977), Friedman (1989), Fujikoshi and Seo (1998), Anderson (2003), Fujikoshi et al. (2010) and many others.

The linear discriminant function is made up of the coefficients called discriminant function coefficients. For the two-group problem the coefficients of the linear discriminant function are taken to be proportional to $\mathbf{S}^{-1}\left(\overline{\mathbf{x}}_{1}-\overline{\mathbf{x}}_{2}\right)$, where $\overline{\mathbf{x}}_{1}, \overline{\mathbf{x}}_{2}$ and $\mathbf{S}$ are the sample mean vectors of the groups and the sample covariance matrix. This function has been studied by Gupta (1968), Rao (1970), Haff (1982), Bodnar and Okhrin (2011) among others. However, their distributional properties and its extended results to large dimension asymptotics have not been intensively considered in the literature. In this thesis we present the product $\mathbf{S}^{-1}\left(\overline{\mathbf{x}}_{1}-\overline{\mathbf{x}}_{2}\right)$ using random variables from standard univariate distributions. We use the stochastic representation to derive the classification function and compute misclassification errors. The results are extended to large dimension asymptotics under the assumption that the dimension $p$ gets close to the sample size $n$ in a way that $p / n$ converges to a positive constant $c \in(0,1)$.

Since the demand for linear discriminant results is not only for cross-sectional data, but also for repeated measures data, the present thesis proposes a linear discriminant function which can classify an individual into one of two populations on the basis of repeated measures observations that have a Potthoff and Roy (1964) growth curve structure on the means. The Growth Curve model which is also known as bilinear regression model is a generalization of the multivariate analysis of variance (MANOVA) model and can be applied to data taken on a subject over $p$ successive time points. Moreover, the standard Growth Curve model first introduced by Potthoff and Roy (1964) assumes that different groups have the same type of profiles. Because, this is clearly not necessarily true, we extend the problem to a more general structue in such a way that the group means follow an Extended Growth Curve model which assumes that different groups follows different growth profiles. In this thesis we also focus on approximating the misclassification probabilities, in particular when the number of repeated measurements is allowed to be large.

The product of the form $\mathbf{S}^{-1}\left(\overline{\mathbf{x}}_{1}-\overline{\mathbf{x}}_{2}\right)$ in the linear discriminant function coefficients appears in portfolio theory introduced by Markowitz (1952) where the vector of optimal portfolio weights is proportional to $\mathbf{S}^{-1} \overline{\mathbf{x}}$. The fundamental goal of the portfolio theory as devised by Markowitz is to determine an efficient way of portfolio allocation. The balance between the variance (risk) and mean (return) of portfolio is at the central part of portfolio theory, which seeks to find optimal allocations of the investor's initial wealth to the available assets. The tangency portfolio (TP) is one such portfolio which consists of both risky and risk-free assets. The sample estimator of the tangency portfolio weights is expressed as a product of an inverse Wishart matrix and a normal random vector. Despite 
the key role of the coefficients in asset allocation, not much has been done from the perspective of the distribution of the portfolio weights. In particular, we provide an analytical result for the higher order moments of the estimated tangency portfolio weights, which also include the expressions for skewness and kurtosis.

\subsection{Aims}

The aim of the present thesis is to contribute to the development of new methods to be used in linear discrimiant analysis with the Growth Curve model. Specific objectives are:

(i) to derive the stochastic representation of the linear discriminant function coefficients and use it to obtain results for classification function in small and large dimensions;

(ii) to propose an approximation of misclassification errors for the linear discriminant function with repeated measurements;

(iii) to derive the approximation for misclassification errors under the Extended Growth Curve model and propose a classification rule with a rank restriction on the mean parameter of the Growth Curve model;

(iv) to derive analytical results for the higher order moments of the estimated tangency portfolio weights.

\subsection{Thesis outline}

This thesis consists of two parts. The first part comprises four chapters where we present necessary concepts for reading the papers which are included in the second part of the thesis.

\subsubsection{Outline of Part I}

The first chapter is the Introduction which consists of the background, aims of the thesis, summary of the papers and contributions. Chapter 2, gives a general introduction to multivariate distributions with main focus on the multivariate normal distribution and the Wishart distribution and their properties. Chapter 3, presents applications of the inverted Wishart matrix and functionals involving both the inverted Wishart matrix and the normal distribution applied to discriminant analysis and the Growth Curve model, and portfolio theory. Part I finalizes with Chapter 4, which gives a summary of contributions and suggestions for further research. 


\title{
1.3.2 Outline of Part II
}

Part II consists of four papers. Below follows a short summary for each of the papers.

\section{Paper A: Discriminant analysis in small and large dimensions}

T. Bodnar, S. Mazur, E. K. Ngailo and N. Parolya. (2019). Discriminant analysis in small and large dimensions. Journal of Theory of Probability and Mathematical Statistics, 100(1), 24-42.

In Paper A, we consider the distributional properties of the linear discriminant function under the assumption of normality where two groups with a common covariance matrix but different mean vectors are compared. The discriminant function coefficients is presented with the help of a stochastic representation which is then used to obtain their asymptotic distribution under a high-dimensional asymptotic setting. We investigate the performance of the classification analysis based on the discriminant function in both small and large dimensions. A stochastic representation s established which allows to compute the error rate in an efficient way. We further compare the calculated error rate with the optimal one obtained under the assumption that the covariance matrix and the two mean vectors are known. Finally, we present an analytical expression of the error rate calculated in a high-dimensional asymptotic regime. The finite-sample properties of the derived theoretical results are assessed via an extensive Monte Carlo study.

\section{Paper B: The approximation for misclassification probabilities in linear discriminant analysis with repeated measurements.}

\author{
E. K. Ngailo, D. von Rosen and M. Singull. (2020). The approximation for \\ misclassification probabilities in linear discriminant analysis with repeated \\ measurements Linköping University Electronic Press LiTH-MAT-R-2020/05-SE.
}

In Paper B we use the linear discriminant function with repeated measurements following the Growth Curve model to derive an approximation of the misclassification errors under the assumption that the covariance matrix is either known and unknown. We assess the performance of the proposed approximation results via a Monte Carlo simulation study.

\section{Paper C: Linear discriminant analysis via the Growth Curve model and restrictions on the mean space}

E. K. Ngailo, D. von Rosen and M. Singull. (2020). Linear discriminant analysis via the Growth Curve model and restrictions on the mean space. Linköping University Electronic Press LiTH-MAT-R-2020/06-SE. 
In Paper C, we consider a linear classification function applied to the Growth Curve model with restrictions on the mean space. Often we must assume the underlying assumption that different groups in the experimental design follow different growth profiles. In this case we have a bilinear restriction on the mean space which leads to an Extended Growth Curve model. Moreover, in this paper we also derive a discriminant function when there exist a rank restriction on the mean parameters.

\section{Paper D: Higher order moments of the estimated tangency portfolio weights}

F. Javed, S. Mazur and E. K. Ngailo. (2020). Higher order moments of the estimated tangency portfolio weights. Accepted for publication in Journal of Applied Statistics: doi.org/10.1080/02664763.2020.1736523

In Paper D we consider the estimated weights of tangency portfolio. We use a stochastic representation of the product of an inverse Wishart matrix and a normal distribution to derive analytical expressions for higher order moments of the weights when the returns are assumed to be independently and multivariate normally distributed. We obtain the expressions for mean, variance, skewness and kurtosis of the estimated weights in closed-forms. Our results are complemented with a simulation study where data from the multivariate normal and $t$-distributions are simulated and the first four moments of the estimated weights are computed by using the Monte Carlo experiment.

\subsection{Author's contributions}

Paper A: Discriminant analysis in small and large dimensions.

Taras Bodnar devised the idea for the project. Edward Ngailo developed theoretical results in cooperation with Taras Bodnar, Stepan Mazur and Nestor Parolya. Edward Ngailo carried out the simulation studies of the developed findings.

Paper B: The approximation for misclassification probabilities in linear discriminant analysis with repeated measurements.

The main conceptual idea of using repeated measurements in discriminant analysis was given by Dietrich von Rosen and Martin Singull. The paper is a result of multiple discussions between Edward Ngailo and the co-authors. Edward Ngailo developed the theoretical framework, made the simulations and wrote largely part of the paper. 
Paper C: Linear discriminant analysis via the Growth Curve model and restrictions on the mean space.

The idea of formulating research problem for Paper $\mathrm{C}$ was suggested by coauthors and Edward Ngailo. The paper is a result of multiple discussions between Edward Ngailo and the co-authors. Edward Ngailo in collaboration with co-authors wrote the paper.

Paper D: Higher order moments of the estimated tangency portfolio weights.

Edward Ngailo contributed in proving the main theorems and carried out the simulation study in collaboration with Stepan Mazur and Farrukh Javed.

Paper A and D were part of Edward Ngailos' licentiate thesis, which was written under supervision of Professor Taras Bodnar at Stockholm University.

Ngailo, E. K. (2018). On functions of a Wishart matrix and a normal vector with applications, Stockholm University: DiVA, id: diva2: 1193512 
Part I

Theoretical background 



\section{Multivariate normal distribution and Wishart distribution}

$\mathrm{T}$ HE multivariate normal distribution has been studied for many years and is the most important multivariate distribution, since many testing, estimation and confidence interval procedures discussed in the multivariate statistical literature are based on assumption that observation vectors are normally distributed, e.g., see Muirhead (1982), Anderson (2003). In this chapter, we give definitions and some results for the normal distribution, Wishart distribution and multivariate linear models.

\subsection{Normal distribution}

Definition 2.1. (Multivariate normal distribution). Let $\boldsymbol{\Sigma}=\boldsymbol{\tau} \boldsymbol{\tau}^{\prime}$, where $\boldsymbol{\tau}: p \times r$. A random vector $\mathbf{x}: p \times 1$ is said to be multivariate normally distributed with parameters $\boldsymbol{\mu}$ and $\Sigma$, if it can be presented in a stochastic representation given by

$$
\mathbf{x} \stackrel{d}{=} \boldsymbol{\mu}+\boldsymbol{\tau} \mathbf{z}
$$

where $\mathbf{z}=\left(z_{1}, \ldots, z_{r}\right)^{\prime}$ and $z_{i} \sim N(0,1)$, with $z_{i}$ and $z_{j}$ independent for $i \neq j$. The sign $\stackrel{d}{=}$ represents "has the same in distribution as".

If the random vector $\mathbf{x}=\left(x_{1}, \ldots, x_{p}\right)^{\prime}: p \times 1$ has a multivariate normal distribution, it is written $\mathbf{x} \sim N_{p}(\boldsymbol{\mu}, \boldsymbol{\Sigma})$, with a mean vector $\boldsymbol{\mu}: p \times 1$ and covariance matrix $\boldsymbol{\Sigma}: p \times p$. If $\boldsymbol{\Sigma}$ is positive definite, then its probability density function is given by

$$
f(\mathbf{x})=(2 \pi)^{-\frac{p}{2}}|\boldsymbol{\Sigma}|^{-\frac{1}{2}} \exp \left\{-\frac{1}{2} \operatorname{tr}\left\{\boldsymbol{\Sigma}^{-1}(\mathbf{x}-\boldsymbol{\mu})(\mathbf{x}-\boldsymbol{\mu})^{\prime}\right\}\right\}, \mathbf{x} \in \mathbb{R}^{p}, \boldsymbol{\mu} \in \mathbb{R}^{p}
$$

where $|\cdot|$ and $\operatorname{tr}(\cdot)$ denote the determinant and the trace of a matrix, respectively. 
To find estimators of the unknown parameters $\boldsymbol{\mu}$ and $\boldsymbol{\Sigma}$, the method of maximum likelihood is often used. In the following we present these estimators.

Example 2.1

Let $\mathbf{x}_{1}, \ldots, \mathbf{x}_{n}$ be independent observations from a multivariate normal distribution $N_{p}(\boldsymbol{\mu}, \boldsymbol{\Sigma})$. Then the likelihood function is given by

$$
\begin{aligned}
L(\boldsymbol{\mu}, \boldsymbol{\Sigma}) & =(2 \pi)^{-\frac{n p}{2}}|\boldsymbol{\Sigma}|^{-\frac{n}{2}} \exp \left\{-\frac{1}{2} \sum_{i=1}^{n}\left(\mathbf{x}_{i}-\boldsymbol{\mu}\right)^{\prime} \boldsymbol{\Sigma}^{-1}\left(\mathbf{x}_{i}-\boldsymbol{\mu}\right)\right\} \\
& =(2 \pi)^{-\frac{n p}{2}}|\boldsymbol{\Sigma}|^{-\frac{n}{2}} \exp \left\{-\frac{1}{2} \operatorname{tr}\left\{\boldsymbol{\Sigma}^{-1}\left(\mathbf{X}-\boldsymbol{\mu} \mathbf{1}_{n}^{\prime}\right)\left(\mathbf{X}-\boldsymbol{\mu} \mathbf{1}_{n}^{\prime}\right)^{\prime}\right\}\right\},
\end{aligned}
$$

where $\mathbf{X}=\left(\mathbf{x}_{1}, \mathbf{x}_{2}, \ldots, \mathbf{x}_{n}\right)$ and $\mathbf{1}_{n}$ is the $n$-dimensional vector of ones. The maximum likelihood estimates of $\boldsymbol{\mu}$ and $\boldsymbol{\Sigma}$ are respectively given by

$$
\widehat{\boldsymbol{\mu}}=\overline{\mathbf{x}}=\frac{1}{n} \sum_{i=1}^{n} \mathbf{x}_{i}=\frac{1}{n} \mathbf{X} \mathbf{1}_{n}, \quad \widehat{\mathbf{\Sigma}}=\frac{1}{n} \mathbf{S},
$$

where

$$
\mathbf{S}=\sum_{i=1}^{n}\left(\mathbf{x}_{i}-\overline{\mathbf{x}}\right)\left(\mathbf{x}_{i}-\overline{\mathbf{x}}\right)^{\prime}=\mathbf{X}\left(\mathbf{I}_{n}-\frac{1}{n} \mathbf{1}_{n} \mathbf{1}_{n}^{\prime}\right) \mathbf{X}^{\prime}
$$

and where $\mathbf{I}_{n}$ denotes the $n \times n$ identity matrix. Note that $\mathbf{I}_{n}-\frac{1}{n} \mathbf{1}_{n} \mathbf{1}_{n}^{\prime}$ is an idempotent matrix.

Now we turn to the definition of the matrix normal distribution.

Definition 2.2. (Matrix normal). A random matrix $\mathbf{X}: p \times n$ is said to be matrix normally distributed with parameters $\mathbf{M}, \boldsymbol{\Sigma}$ and $\boldsymbol{\Psi}$, if it can be presented in a stochastic representation given by

$$
\mathbf{X} \stackrel{d}{=} \mathbf{M}+\tau \mathbf{U} \gamma^{\prime}
$$

where $\mathbf{U}: r \times s$ is a random matrix which consists of $s$ independent and identically distributed $N_{r}\left(\mathbf{0}_{r}, \mathbf{I}\right)$ vectors $\mathbf{U}_{i}, i=1,2, \ldots, s, \boldsymbol{\Sigma}=\boldsymbol{\tau} \boldsymbol{\tau}^{\prime}$ and $\boldsymbol{\Psi}=\boldsymbol{\gamma} \boldsymbol{\gamma}^{\prime}, \boldsymbol{\tau}$ is $p \times r$ and $\gamma$ is $n \times s$. Here $\mathbf{0}_{r}$ is the $r$-dimensional vector of zeros.

If a random matrix $\mathbf{X}: p \times n$ is matrix normally distributed with mean $\mathbf{M}: p \times n$ and positive definite covariance matrices $\boldsymbol{\Sigma}: p \times p$ and $\boldsymbol{\Psi}: n \times n$ it has the density function

$$
f(\mathbf{X})=(2 \pi)^{-\frac{p n}{2}}|\boldsymbol{\Sigma}|^{-\frac{n}{2}}|\boldsymbol{\Psi}|^{-\frac{p}{2}} \exp \left\{-\frac{1}{2} \operatorname{tr}\left\{\boldsymbol{\Sigma}^{-1}(\mathbf{X}-\mathbf{M}) \boldsymbol{\Psi}^{-1}(\mathbf{X}-\mathbf{M})^{\prime}\right\}\right\} .
$$

The matrix normally distribution of $\mathbf{X}$ is in this thesis denoted $\mathbf{X} \sim N_{p, n}(\mathbf{M}, \boldsymbol{\Sigma}, \mathbf{\Psi})$. 


\subsection{Wishart distribution}

\subsubsection{Wishart and non-central Wishart distribution}

The Wishart distribution is a multivariate generalization of the chi-square distribution (Fujikoshi et al. (2010), Rencher and Christensen (2012)). It is named in honor of John Wishart, who first formulated the distribution in 1928.

Definition 2.3. (Wishart distribution). A random matrix $\mathbf{S}: p \times p$ is said to be Wishart distributed if and only if $\mathbf{S}=\mathbf{X X}^{\prime}$ for some $p \times n$ matrix $\mathbf{X}$, where $\mathbf{X} \sim N_{p, n}(\mathbf{M}, \boldsymbol{\Sigma}, \mathbf{I})$. If the mean is zero, that is $\mathbf{M}=\mathbf{0}$, the Wishart distribution is said to be central Wishart distributed, which is denoted by $\mathbf{S} \sim W_{p}(n, \boldsymbol{\Sigma})$. If the mean $\mathbf{M} \neq \mathbf{0}$, the resulting distribution is non-central Wishart distributed and is denoted $\mathbf{S} \sim W_{p}(n, \boldsymbol{\Sigma}, \mathbf{\Omega})$, where $\Omega=\mathrm{MM}^{\prime}$.

The first parameter $n$ stands for the degrees of freedom and is usually considered to be known. The second parameter $\boldsymbol{\Sigma}$ is usually supposed to be unknown, the third parameter $\boldsymbol{\Omega}$ stands for the non-centrality parameter. When $\boldsymbol{\Sigma}>0$ (positive definite) and $n \geq p$, the density function for $\mathbf{S} \sim W_{p}(n, \boldsymbol{\Sigma})$ exists and is given by

$$
f_{\mathbf{S}}(\mathbf{S})=c(p, n)^{-1}|\boldsymbol{\Sigma}|^{-\frac{n}{2}}|\mathbf{S}|^{\frac{(n-p-1)}{2}} \exp \left\{-\frac{1}{2} \operatorname{tr}\left\{\boldsymbol{\Sigma}^{-1} \mathbf{S}\right\}\right\},
$$

for $\mathbf{S}>0$, and 0 otherwise, where $c(p, n)$ is the normalizing constant given by

$$
c(p, n)=2^{\frac{p n}{2}} \pi^{\frac{1}{4} p(p-1)} \prod_{i=1}^{p} \Gamma\left(\frac{1}{2}(n+1-i)\right),
$$

and $\Gamma(\cdot)$ is the gamma function. If $n<p$, then $\mathbf{S}$ is singular and the $\mathbf{S} \sim W_{p}(n, \boldsymbol{\Sigma})$ distribution does not have a density function.

In the following theorems we give some useful properties for the Wishart and inverted Wishart distributions. The theorems are useful when proving results in Section 2.1 in Paper A and some results in Paper D. The proofs of the results of theorems can be found, for example in Muirhead (1982), Gupta and Nagar (2000) and Kollo and von Rosen (2005).

\section{Theorem 2.1}

Let $\mathbf{S} \sim W_{p}(n, \boldsymbol{\Sigma}, \boldsymbol{\Omega})$ and let $\mathbf{A}$ be a $q \times p$ matrix. Then

$$
\mathbf{A S A}^{\prime} \sim W_{q}\left(n, \mathbf{A} \boldsymbol{\Sigma} \mathbf{A}^{\prime}, \mathbf{A} \boldsymbol{\Omega} \mathbf{A}^{\prime}\right) .
$$

The sum of independent Wishart distributed variables is again Wishart distributed and can easily be seen from the definition of a Wishart distribution.

\section{Theorem 2.2}

Let $\mathbf{S}_{1}$ and $\mathbf{S}_{2}$ be independent with $\mathbf{S}_{i} \sim W_{p}\left(n_{i}, \boldsymbol{\Sigma}, \boldsymbol{\Omega}_{i}\right), i=1,2$. Then

$$
\mathbf{S}_{1}+\mathbf{S}_{2} \sim W_{p}\left(n_{1}+n_{2}, \boldsymbol{\Sigma}, \boldsymbol{\Omega}_{1}+\boldsymbol{\Omega}_{2}\right) .
$$




\section{Theorem 2.3}

Let $\mathbf{S} \sim W_{p}(n, \mathbf{\Sigma})$, where $n>p-1$, and $\mathbf{x}$ is any $p \times 1$ random vector distributed independently of $\mathbf{S}$ with $\mathbf{x} \neq \mathbf{0}$ almost surely. Then

$$
\frac{\mathbf{x}^{\prime} \boldsymbol{\Sigma}^{-1} \mathbf{x}}{\mathbf{x}^{\prime} \mathbf{S}^{-1} \mathbf{x}} \sim \chi_{n-p+1}^{2},
$$

which is independent of $\mathbf{x}$.

Trivially the conditional distribution of $\frac{\mathbf{x}^{\prime} \boldsymbol{\Sigma}^{-1} \mathbf{x}}{\mathbf{x}^{\prime} \mathbf{S}^{-1} \mathbf{x}}$ given $\mathbf{x}$ is $\chi_{n-p+1}^{2}$.

\section{Corollary 2.1}

Let $\overline{\mathbf{x}}$ and $\mathbf{S}$ be defined as in Example 2.1, then

$$
\frac{\overline{\mathbf{x}}^{\prime} \boldsymbol{\Sigma}^{-1} \overline{\mathbf{x}}}{\overline{\mathbf{x}}^{\prime} \mathbf{S}^{-1} \overline{\mathbf{x}}} \sim \chi_{n-p}^{2},
$$

and is independent of $\overline{\mathbf{x}}$.

\subsubsection{Moments of the inverted Wishart distribution}

In the following theorem we consider the moments for $\mathbf{S}^{-1}$ and functions involving $\mathbf{S}^{-1}$. Let $\mathbb{E}[\cdot]$ denote the expectation.

\section{Theorem 2.4}

Let $\mathbf{S} \sim W_{p}(n, \boldsymbol{\Sigma})$ and let $\mathbf{A}$ be a $p \times q$ matrix of constants $\operatorname{rank}(\mathbf{A})=q$. Then

(i) $\mathbb{E}\left[\mathbf{S}^{-1}\right]=d_{1} \boldsymbol{\Sigma}^{-1}$,

(ii) $\mathbb{E}\left[\mathbf{S}^{-1} \mathbf{\Sigma} \mathbf{S}^{-1}\right]=(n-1) d_{2} \boldsymbol{\Sigma}^{-1}$,

(iii) $\mathbb{E}\left[\mathbf{S}^{-1} \mathbf{A}\left(\mathbf{A}^{\prime} \mathbf{S}^{-1} \mathbf{A}\right)^{-1} \mathbf{A}^{\prime} \mathbf{S}^{-1} \mathbf{\Sigma} \mathbf{S}^{-1} \mathbf{A}\left(\mathbf{A}^{\prime} \mathbf{S}^{-1} \mathbf{A}\right)^{-1} \mathbf{A}^{\prime} \mathbf{S}^{-1}\right]$

$$
=(n-1) d_{2} \boldsymbol{\Sigma}^{-1}+\left[(n-1) d_{3}-(n+p-2 q-1) d_{4}\right]\left(\boldsymbol{\Sigma}^{-1}-\boldsymbol{\Sigma}^{-1} \mathbf{A}\left(\mathbf{A}^{\prime} \boldsymbol{\Sigma} \mathbf{A}\right)^{-1} \mathbf{A}^{\prime} \boldsymbol{\Sigma}^{-1}\right),
$$

where

$$
\begin{array}{lrl}
d_{1}=\frac{1}{n-p-1}, & \text { if } n-p-1>0, \\
d_{2}=\frac{1}{(n-p)(n-p-1)(n-p-3)}, & \text { if } n-p-3>0, \\
d_{3}=\frac{1}{(n-(p-q))(n-(p-q)-1)(n-(p-q)-3)}, & \text { if } n>(p-q)-3, \\
d_{4}=\frac{1}{(n-q)(n-(p-q)-1)(n-q-3)}, & \text { if } n-q>0, n>(p-q)-1 .
\end{array}
$$

The proofs and technical derivations of these results are found, for example, in Gupta (1968), von Rosen (1988), Gupta and Nagar (2000) and von Rosen (2018). Statement (i) is 
useful in Paper A [Section 2.1 and 2.3], and statements (i) through (iii) are useful in Paper B [Section 3] for calculating the moments in the case when $\Sigma$ is unknown. Statement (i) is also applied in Paper D for obtaining moments when deriving results for the tangency portfolio weights.

\subsection{The Growth Curve model}

The Growth Curve model is a generalization of the multivariate analysis of variance (MANOVA) model and can be applied to model repeated measures data with a mean structure which for example is polynomial in time. The classical MANOVA model is defined by

$$
\mathbf{X}=\mathbf{B C}+\mathbf{E}
$$

where $\mathbf{X}: p \times n, \mathbf{B}: p \times k$ and $\mathbf{C}: k \times n$ are the observations, unknown parameter and known design matrices respectively. It is assumed that $\mathbf{E}: p \times n$ is distributed as multivariate normal distribution with mean $\mathbf{0}$ and positive definite covariance matrix $\boldsymbol{\Sigma}$, that is, $\mathbf{E} \sim N_{p, n}\left(\mathbf{0}, \boldsymbol{\Sigma}, \mathbf{I}_{n}\right)$. When $n \geq \operatorname{rank}(\mathbf{C})+p$ and $\mathbf{C}$ is assumed to have full rank, the maximum likelihood estimators for $\mathbf{B}$ and $\boldsymbol{\Sigma}$ are given by

$$
\widehat{\mathbf{B}}=\mathbf{X C}^{\prime}\left(\mathbf{C C}^{\prime}\right)^{-1}, n \widehat{\mathbf{\Sigma}}=\mathbf{X} \mathbf{Q}_{\mathbf{C}^{\prime}} \mathbf{X}^{\prime}
$$

where $\mathbf{Q}_{\mathbf{C}^{\prime}}=\mathbf{I}-\mathbf{P}_{\mathbf{C}^{\prime}}, \mathbf{P}_{\mathbf{C}^{\prime}}=\mathbf{C}^{\prime}\left(\mathbf{C C}^{\prime}\right)^{-1} \mathbf{C}$ is the projection matrix onto the column space $\mathcal{C}\left(\mathbf{C}^{\prime}\right)$ and $\mathcal{C}(\cdot)$ represents the column space of a matrix.

The Growth Curve model is an extension of the MANOVA model (2.2) and also known as the generalized multivariate analysis of variance (GMANOVA) model. Let $\mathbf{X}: p \times n$ be the observation matrix and $\mathbf{B}: q \times k$ be the unknown growth curve parameter matrix and let $\mathbf{A}: p \times q$ and $q \leq p, \mathbf{C}: k \times n$, of $\operatorname{rank}(\mathbf{C}) \leq n-p$ be the within and between individual design matrices, respectively. Then, the Growth Curve model is given by

$$
\mathbf{X}=\mathbf{A B C}+\mathbf{E}
$$

where $\mathbf{E} \sim N_{p, n}\left(\mathbf{0}, \boldsymbol{\Sigma}, \mathbf{I}_{n}\right)$ and $\boldsymbol{\Sigma}$ is unknown. If assuming a polynomial growth of order $q-1$ and $k$ independent treatments groups, each comprising $n_{i}$ individuals the two design matrices $\mathbf{A}$ and $\mathbf{C}$ are given by

$$
\mathbf{A}=\left(\begin{array}{cccc}
1 & t_{1} & \ldots & t_{1}^{q-1} \\
1 & t_{2} & \ldots & t_{2}^{q-1} \\
\vdots & \vdots & \ddots & \vdots \\
1 & t_{p} & \ldots & t_{p}^{q-1}
\end{array}\right), \mathbf{C}=\left(\begin{array}{cccc}
\mathbf{1}_{n_{1}}^{\prime} & \mathbf{0}_{n_{1}}^{\prime} & \ldots & \mathbf{0}_{n_{1}}^{\prime} \\
\mathbf{0}_{n_{2}}^{\prime} & \mathbf{1}_{n_{2}}^{\prime} & \ldots & \mathbf{0}_{n_{2}}^{\prime} \\
\vdots & \vdots & \ddots & \vdots \\
\mathbf{0}_{n_{k}}^{\prime} & \mathbf{0}_{n_{k}}^{\prime} & \ldots & \mathbf{1}_{n_{k}}^{\prime}
\end{array}\right), \mathbf{B}=\left(\mathbf{b}_{1}, \mathbf{b}_{2}, \ldots, \mathbf{b}_{k}\right)
$$


Note that the matrix $\mathbf{A}$ models the within-individual structure, whereas $\mathbf{C}$ models the between-individual structure. In particular, the within-individual design matrix $\mathbf{A}$ contains time regressors and models growth profiles, and the between-individual design matrix $\mathbf{C}$ is composed of group separation indicators.

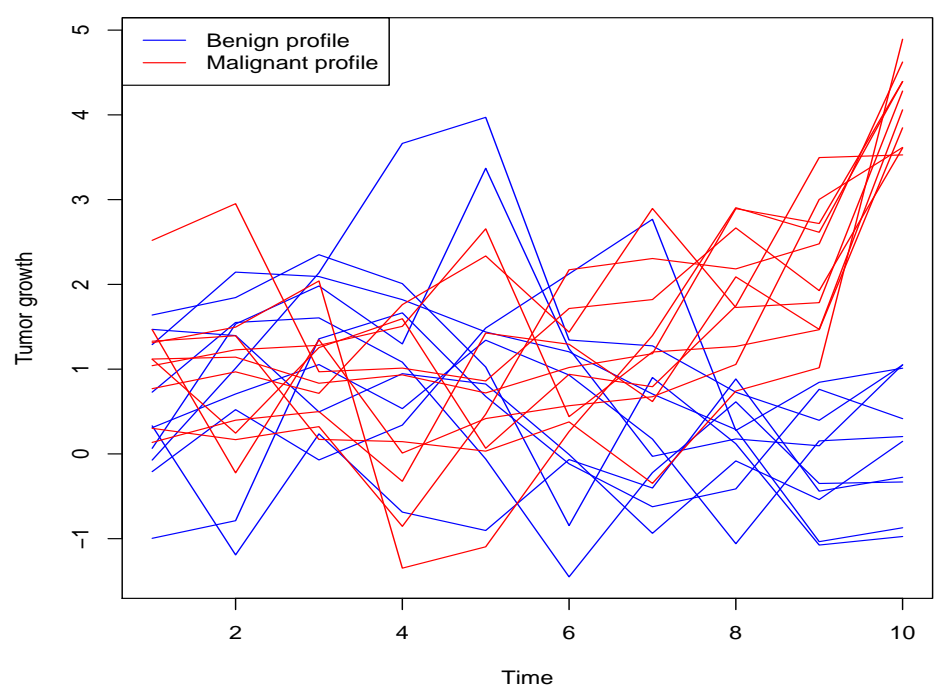

Figure 2.1: Growth profiles for the benign tumor showing decrease in tumor sizes and malignant tumor showing increase in tumor sizes.

\section{Example 2.2: Benign and Malignant tumor}

There are two general types of tumors: benign (non-cancerous) tumors and malignant (cancerous) tumors. The benign and malignant tumor simulated data set listed in Table 2.1 can be assumed to follow the Growth Curve model (2.3). Suppose third order growth curves describe the growth profiles for the benign $(i=1)$ and malignant $(i=2)$ tumors as

$$
\mu_{i}=b_{0 i}+b_{1 i} t+b_{2 i} t^{2}+b_{3 i} t^{3}, \quad i=1,2 .
$$

Moreover, assume that we have a possibility to perform $p=10$ repeated measurements on tumor size during 10 time units, i.e., $t=1, \ldots, 10$. Then the design matrices $\mathbf{A}$ and $\mathbf{C}$ and the parameter matrix $\mathbf{B}$ are given by 
Table 2.1: Tumor repeated measurements for 10 benign tumor and 10 malignant tumor.

\begin{tabular}{|c|c|c|c|c|c|c|c|c|c|c|c|}
\hline \multicolumn{12}{|c|}{ Individuals } \\
\hline Benign & & 1 & 2 & 3 & 4 & 5 & 6 & 7 & 8 & 9 & 10 \\
\hline \multirow{10}{*}{ Time } & 1 & -0.07 & 0.31 & 1.29 & 1.64 & 1.47 & -1.00 & 0.33 & 0.73 & 0.07 & -0.21 \\
\hline & 2 & 1.01 & 0.71 & 2.14 & 1.84 & 1.40 & -0.79 & -1.19 & 1.55 & 1.53 & 0.52 \\
\hline & 3 & 2.14 & 1.05 & 2.09 & 2.35 & 0.50 & 1.36 & 0.24 & 1.60 & 1.98 & -0.07 \\
\hline & 4 & 3.66 & 0.53 & 1.82 & 2.01 & 0.95 & 1.66 & -0.69 & 1.08 & 1.30 & 0.34 \\
\hline & 5 & 3.97 & 1.34 & 1.44 & 1.03 & 0.82 & 0.68 & -0.90 & -0.06 & 3.37 & 1.49 \\
\hline & 6 & 1.34 & 0.93 & 1.20 & -0.85 & -0.01 & -0.12 & -0.07 & -1.45 & 1.24 & 2.12 \\
\hline & 7 & 1.27 & 0.17 & 0.71 & 0.90 & -0.94 & -0.62 & -0.40 & -0.22 & -0.03 & 2.77 \\
\hline & 8 & 0.73 & -1.06 & 0.29 & 0.12 & -0.08 & -0.41 & 0.88 & 0.61 & 0.18 & 0.28 \\
\hline & 9 & 0.40 & 0.15 & -1.04 & -1.08 & -0.54 & 0.76 & -0.44 & -0.35 & 0.10 & 0.84 \\
\hline & 10 & 1.05 & 0.20 & -0.87 & -0.97 & 0.15 & 0.42 & -0.28 & -0.33 & 1.05 & 1.01 \\
\hline \multirow[t]{5}{*}{ Malignant } & & 1 & 2 & 3 & 4 & 5 & 6 & 7 & 8 & 9 & 10 \\
\hline & 1 & 1.31 & 1.04 & 0.77 & 1.33 & 0.30 & 1.12 & 2.52 & 1.47 & 0.14 & 1.12 \\
\hline & 2 & 1.50 & 1.23 & 0.97 & 1.39 & 0.17 & 0.24 & 2.95 & -0.22 & 0.40 & 1.14 \\
\hline & 3 & 2.04 & 1.28 & 0.71 & 0.17 & 0.32 & 1.25 & 0.97 & 1.35 & 0.50 & 0.83 \\
\hline & 4 & -1.35 & 1.51 & 1.76 & 0.14 & -0.85 & 1.60 & 1.01 & 0.01 & -0.32 & 0.93 \\
\hline \multirow[t]{6}{*}{ Time } & 5 & -1.10 & 2.66 & 2.34 & 0.03 & 0.47 & 0.07 & 0.86 & 0.42 & 1.43 & 0.72 \\
\hline & 6 & 0.26 & 0.44 & 1.44 & 0.38 & 2.17 & 0.94 & 1.72 & 0.57 & 1.29 & 1.02 \\
\hline & 7 & 1.40 & 1.20 & 2.90 & -0.35 & 2.31 & 0.79 & 1.82 & 0.67 & 0.62 & 1.18 \\
\hline & 8 & 2.90 & 1.27 & 1.73 & 0.74 & 2.18 & 1.75 & 2.67 & 1.06 & 2.09 & 2.89 \\
\hline & 9 & 2.61 & 1.47 & 1.78 & 1.02 & 2.48 & 3.50 & 1.93 & 3.00 & 1.47 & 2.72 \\
\hline & 10 & 4.39 & 3.85 & 4.28 & 4.89 & 4.62 & 3.53 & 3.61 & 3.62 & 4.06 & 4.39 \\
\hline
\end{tabular}

$$
\mathbf{A}=\left(\begin{array}{cccc}
1 & 1 & 1 & 1 \\
1 & 2 & 4 & 8 \\
\vdots & \vdots & \vdots & \vdots \\
1 & 10 & 100 & 1000
\end{array}\right), \quad \mathbf{C}=\left(\begin{array}{cc}
\mathbf{1}_{10}^{\prime} & \mathbf{0}_{10}^{\prime} \\
\mathbf{0}_{10}^{\prime} & \mathbf{1}_{10}^{\prime}
\end{array}\right), \quad \mathbf{B}=\left(\begin{array}{ll}
b_{01} & b_{02} \\
b_{11} & b_{12} \\
b_{21} & b_{22} \\
b_{31} & b_{32}
\end{array}\right)
$$

Figure 2.1 illustrates the growth profiles of tumor sizes for both benign and malignant tumors for a period of 10 time units.

Estimation of the parameters in the model (2.3) was studied by Khatri (1966) using the likelihood function. The book by von Rosen (2018) contains detailed information on estimation of the maximum likelihood estimators. If $\mathbf{A}$ and $\mathbf{C}$ are of full rank, the 
maximum likelihood estimator of the parameter matrix $\mathbf{B}$ is given by

$$
\widehat{\mathbf{B}}=\left(\mathbf{A}^{\prime} \mathbf{S}^{-1} \mathbf{A}\right)^{-1} \mathbf{A}^{\prime} \mathbf{S}^{-1} \mathbf{X} \mathbf{C}^{\prime}\left(\mathbf{C} \mathbf{C}^{\prime}\right)^{-1},
$$

where $\mathbf{S}$ represents the sum of squares matrix and is given by

$$
\mathbf{S}=\mathbf{X}\left(\mathbf{I}-\mathbf{P}_{\mathbf{C}^{\prime}}\right) \mathbf{X}^{\prime}, \quad \mathbf{P}_{\mathbf{C}^{\prime}}=\mathbf{C}^{\prime}\left(\mathbf{C C}^{\prime}\right)^{-1} \mathbf{C} .
$$

The maximum likelihood estimator for the covariance matrix $\Sigma$ is given by

$$
n \widehat{\mathbf{\Sigma}}=(\mathbf{X}-\mathbf{A B} \mathbf{C})(\mathbf{X}-\mathbf{A} \widehat{\mathbf{B}} \mathbf{C})^{\prime}
$$

\section{Example 2.3: Example 2.2 continued}

Consider again the benign and malignant simulated tumor data set (Table 2.1) and the model in (2.3). Then, the maximum likelihood estimates of the parameters equal

$$
\widehat{\mathbf{B}}=\left(\begin{array}{cc}
-0.144 & 1.001 \\
0.907 & 0.387 \\
-0.192 & -0.153 \\
0.010 & 0.014
\end{array}\right), \quad \widehat{\boldsymbol{\Sigma}}=\left(\begin{array}{cccc}
0.523 & 0.491 & \ldots & -0.259 \\
0.491 & 0.968 & \ldots & -0.097 \\
\vdots & \vdots & \ddots & \vdots \\
-0.259 & -0.097 & \ldots & 0.358
\end{array}\right)
$$

Hence, the estimated mean growth curves, plotted in Figure 2.2, for benign $(b)$ and malignant $(m)$ are respectively

$$
\begin{aligned}
& \hat{\mu}_{b}=-0.144+0.907 t-0.192 t^{2}+0.010 t^{3}, \\
& \hat{\mu}_{m}=1.001+0.387 t-0.153 t^{2}+0.014 t^{3} .
\end{aligned}
$$

In Figure 2.2 the sample means per group (solid red and solid blue lines) and the estimated (dotted red and dotted blue curves) growth profiles for both the benign and the malignant tumors are presented. In Figure 2.2 we observe that the malignant growth curves grow. This means there is an increase in malignant tumor size with time. On the other hand the benign tumor show a negative growth and takes small values. This means that benign tumor decreases in size with time.

The Growth Curve model given in (2.3), assumes that all the treatment groups should follow the same growth profile. This assumption is not necessarily true and therefore the Extended Growth Curve model (EGCM) is considered which is given by

$$
\mathbf{X}=\sum_{i=1}^{m} \mathbf{A}_{i} \mathbf{B}_{i} \mathbf{C}_{i}+\mathbf{E}
$$




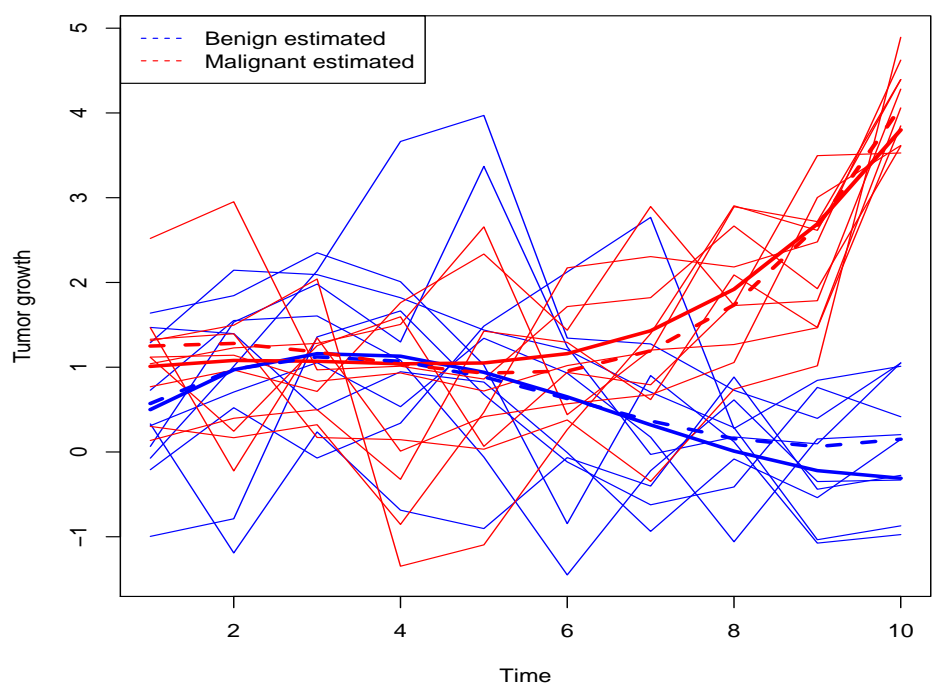

Figure 2.2: The sample means per group (solid lines) and the estimated growth profiles (dotted lines) for the benign and malignnant tumors.

where $\mathbf{X}: p \times n, \mathbf{A}_{i}: p \times q_{i}, \mathbf{B}_{i}: q_{i} \times k_{i}, \mathbf{C}_{i}: k_{i} \times n, p \leq n-\operatorname{rank}\left(\mathbf{C}_{1}\right), i=$ $1,2, \ldots, m, \mathcal{C}\left(\mathbf{C}_{i}^{\prime}\right) \subseteq \mathcal{C}\left(\mathbf{C}_{i-1}^{\prime}\right), i=1,2,3, \ldots, m$, the columns of $\mathbf{E}$ are assumed to be independently distributed as multivariate normal with zero mean and a positive definite dispersion matrix $\boldsymbol{\Sigma}$; i.e., $\mathbf{E} \sim N_{p, n}\left(\mathbf{0}, \boldsymbol{\Sigma}, \mathbf{I}_{n}\right)$. The matrices $\mathbf{A}_{i}$ and $\mathbf{C}_{i}$ are known design matrices whereas $\mathbf{B}_{i}$ and $\boldsymbol{\Sigma}$ are unknown parameter matrices.

The only difference with the Growth Curve model given in (2.3) is the presence of a more general mean structure. The model without restrictions on the subspaces was studied by Verbyla and Venables (1988) under the name sum of profiles model. The model was also studied by von Rosen (1989) who gave explicit form of the maximum likelihood estimators under the nested subspace condition between the within-individuals design matrices, that is, $\mathcal{C}\left(\mathbf{C}_{i}^{\prime}\right) \subseteq \mathcal{C}\left(\mathbf{C}_{i-1}^{\prime}\right), i=2,3, \ldots, m$. The EGCM for two different growth profiles $(m=2)$ is given by

$$
\mathbf{X}=\mathbf{A}_{1} \mathbf{B}_{1} \mathbf{C}_{1}+\mathbf{A}_{2} \mathbf{B}_{2} \mathbf{C}_{2}+\mathbf{E}, \quad \mathbf{E} \sim N_{p, n}(\mathbf{0}, \boldsymbol{\Sigma}, \mathbf{I}), \mathcal{C}\left(\mathbf{C}_{2}^{\prime}\right) \subseteq \mathcal{C}\left(\mathbf{C}_{1}^{\prime}\right),
$$


where for example

$$
\begin{aligned}
& \mathbf{A}_{1}=\left(\begin{array}{cccc}
1 & t_{1} & \ldots & t_{1}^{q-2} \\
1 & t_{2} & \ldots & t_{2}^{q-2} \\
\vdots & \vdots & \ddots & \vdots \\
1 & t_{p} & \ldots & t_{p}^{q-2}
\end{array}\right), \mathbf{A}_{2}=\left(\begin{array}{c}
t_{1}^{q-1} \\
t_{2}^{q-1} \\
\ldots \\
t_{p}^{q-1}
\end{array}\right), \\
& \mathbf{B}_{1}=\left(\begin{array}{cc}
b_{01} & b_{02} \\
b_{11} & b_{12} \\
\vdots & \vdots \\
b_{(q-1) 1} & b_{(q-1) 2}
\end{array}\right), \mathbf{B}_{2}=\left(b_{q 2}\right), \\
& \mathbf{C}_{1}=\left(\begin{array}{ll}
\mathbf{1}_{n_{1}}^{\prime} & \mathbf{0}_{n_{1}}^{\prime} \\
\mathbf{0}_{n_{2}}^{\prime} & \mathbf{1}_{n_{2}}^{\prime}
\end{array}\right), \mathbf{C}_{2}=\left(\begin{array}{ll}
\mathbf{0}_{n_{1}}^{\prime} & \mathbf{1}_{n_{2}}^{\prime}
\end{array}\right) .
\end{aligned}
$$

It should be noted that the Extended Growth Curve model can be viewed as a Growth Curve model with restrictions on the mean parameter. In a matrix language one can write the Extended Growth Curve model as $E[\mathbf{X}]=\mathbf{A B C}$ with the restriction $\mathbf{F B G}=\mathbf{0}$, where $\mathbf{F}$ and $\mathbf{G}$ are known matrices.

Instead of the subspace condition $\mathcal{C}\left(\mathbf{C}_{i}^{\prime}\right) \subseteq \mathcal{C}\left(\mathbf{C}_{i-1}^{\prime}\right), i=2,3, \ldots, m$, Filipiak and von Rosen (2012) showed that an equivalent model can be given with the subspace condition $\mathcal{C}\left(\mathbf{A}_{i-1}\right) \subseteq \mathcal{C}\left(\mathbf{A}_{i}\right), i=2,3, \ldots, m$. These conditions lead to a different parameterizations and the particular model is given by

$$
\mathbf{X}=\mathbf{A}_{1} \mathbf{B}_{1} \mathbf{C}_{1}+\mathbf{A}_{2} \mathbf{B}_{2} \mathbf{C}_{2}+\mathbf{E}, \quad \mathbf{E} \sim \mathbf{N}_{p, n}(\mathbf{0}, \boldsymbol{\Sigma}, \mathbf{I}), \quad \mathcal{C}\left(\mathbf{A}_{1}\right) \subseteq \mathcal{C}\left(\mathbf{A}_{2}\right),
$$

where for instance the above example can be converted to (2.7) through

$$
\begin{aligned}
\mathbf{A}_{1}= & \left(\begin{array}{cccc}
1 & t_{1} & \ldots & t_{1}^{q-2} \\
1 & t_{2} & \ldots & t_{2}^{q-2} \\
\vdots & \vdots & \ddots & \vdots \\
1 & t_{p} & \ldots & t_{p}^{q-2}
\end{array}\right), \quad \mathbf{A}_{2}=\left(\begin{array}{cccc}
1 & t_{1} & \ldots & t_{1}^{q-1} \\
1 & t_{2} & \ldots & t_{2}^{q-1} \\
\vdots & \vdots & \ddots & \vdots \\
1 & t_{p} & \ldots & t_{p}^{q-1}
\end{array}\right) \\
\mathbf{B}_{1}= & \left(\begin{array}{c}
b_{12} \\
b_{22} \\
\vdots \\
b_{(q-1) 2}
\end{array}\right), \quad \mathbf{B}_{2}=\left(\begin{array}{c}
b_{11} \\
b_{21} \\
\vdots \\
b_{q 2}
\end{array}\right)
\end{aligned}
$$

and $\mathbf{C}_{1}=\left(\mathbf{1}_{n_{1}}^{\prime}: \mathbf{0}_{n_{2}}^{\prime}\right): 1 \times n_{1}, \mathbf{C}_{2}=\left(\mathbf{0}_{n_{1}}^{\prime}: \mathbf{1}_{n_{2}}^{\prime}\right): 1 \times n_{2}$. Note that, in model (2.7), the first and second mean terms, $\mathbf{A}_{1} \mathbf{B}_{1} \mathbf{C}_{1}$ and $\mathbf{A}_{2} \mathbf{B}_{2} \mathbf{C}_{2}$, have two different growth 
profiles. Assuming $\boldsymbol{\Sigma}$ to be known, the maximum likelihood estimators are given by

$$
\begin{aligned}
& \widehat{\mathbf{B}}_{1}=\left(\mathbf{A}_{1}^{\prime} \boldsymbol{\Sigma}^{-1} \mathbf{A}_{1}\right)^{-1} \mathbf{A}_{1}^{\prime} \boldsymbol{\Sigma}^{-1} \frac{1}{n_{1}} \mathbf{X}^{(1)} \mathbf{1}_{n_{1}}=\left(\mathbf{A}_{1}^{\prime} \boldsymbol{\Sigma}^{-1} \mathbf{A}_{1}\right)^{-1} \mathbf{A}_{1}^{\prime} \boldsymbol{\Sigma}^{-1} \overline{\mathbf{x}}_{1}, \\
& \widehat{\mathbf{B}}_{2}=\left(\mathbf{A}_{2}^{\prime} \boldsymbol{\Sigma}^{-1} \mathbf{A}_{2}\right)^{-1} \mathbf{A}_{2}^{\prime} \boldsymbol{\Sigma}^{-1} \frac{1}{n_{2}} \mathbf{X}^{(2)} \mathbf{1}_{n_{2}}=\left(\mathbf{A}_{2}^{\prime} \boldsymbol{\Sigma}^{-1} \mathbf{A}_{2}\right)^{-1} \mathbf{A}_{2}^{\prime} \boldsymbol{\Sigma}^{-1} \overline{\mathbf{x}}_{2} .
\end{aligned}
$$

The handling of $\widehat{\mathbf{B}}_{1}$ and $\widehat{\mathbf{B}}_{2}$ in discriminant analysis when $\boldsymbol{\Sigma}$ is unknown, is much more complicated. It will be treated in subsequent research.

\section{Example 2.4: Example 2.2 continued}

Consider the benign and malignant simulated tumor data. Then we may use the Extended Growth Curve model in (2.7) where the design matrices and parameters are given by

$$
\begin{aligned}
& \mathbf{A}_{1}=\left(\begin{array}{cccc}
1 & 1 & 1 & 1 \\
1 & 2 & 2^{2} & 2^{3} \\
\vdots & \vdots & \vdots & \vdots \\
1 & 10 & 10^{2} & 10^{3}
\end{array}\right), \mathbf{C}_{1}=\left(\mathbf{1}_{10}^{\prime}: \mathbf{0}_{10}^{\prime}\right) \\
& \mathbf{A}_{2}=\left(\begin{array}{ccccc}
1 & 1 & 1 & 1 & 1 \\
1 & 2 & 2^{2} & 2^{3} & 2^{4} \\
\vdots & \vdots & \vdots & \vdots & \vdots \\
1 & 10 & 10^{2} & 10^{3} & 10^{4}
\end{array}\right), \mathbf{C}_{2}=\left(\mathbf{0}_{10}^{\prime}: \mathbf{1}_{10}^{\prime}\right), \\
& \mathbf{B}_{1}=\left(b_{01}, b_{11}, b_{21}, b_{31}\right)^{\prime}, \mathbf{B}_{2}=\left(b_{02}, b_{12}, b_{22}, b_{32}, b_{42}\right)^{\prime} .
\end{aligned}
$$

The within-individual design matrix $\mathbf{A}_{1}$ models the third order polynomial growth curve for the benign tumor group and $\mathbf{A}_{2}$ models the fourth order polynomial growth curve for malignant tumor group. The maximum likelihood estimators of the parameters equal

$$
\widehat{\mathbf{B}}_{1}=(-0.642,1.333,-0.275,0.015)^{\prime}, \widehat{\mathbf{B}}_{2}=(1.744,-0.865,0.288,-0.042,0.003)^{\prime} .
$$

It can be observed in Figure 2.3 that the tumor size for the benign tumors (dotted and solid blue curves) decreases in size with time, whereas the malignant tumor size (dotted and solid red curves) increases with time. 


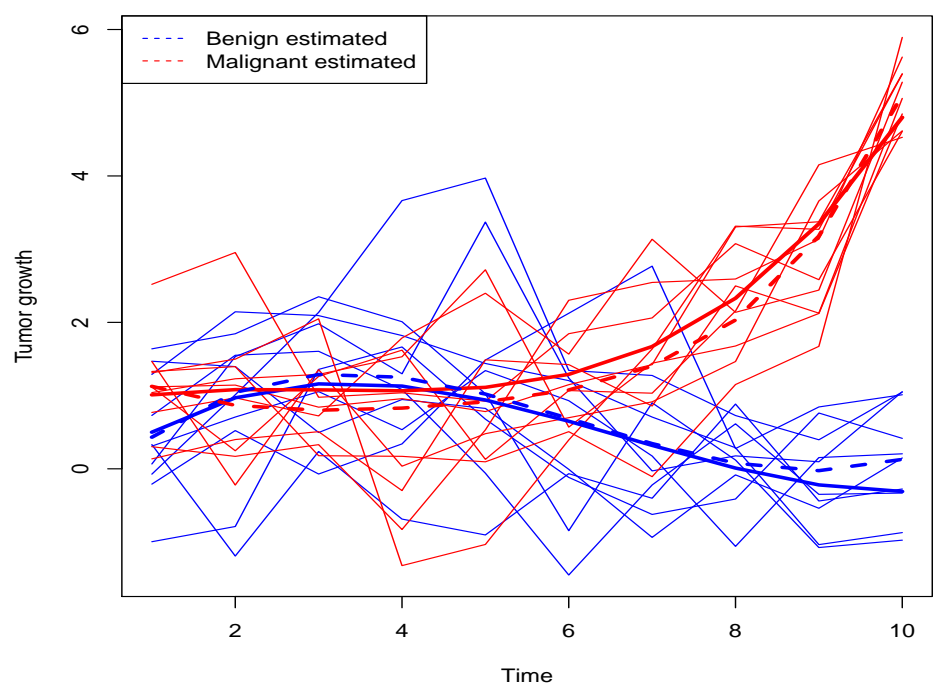

Figure 2.3: The sample means per group for the benign and malignant growth profiles (solid lines) and the estimated growth profiles for the benign and malignant tumors (dotted lines) in the Extended Growth Curve model. 


\title{
Discriminant analysis
}

\begin{abstract}
$\mathrm{T}$ HE goal of this chapter is to give definitions and some results on discriminant analysis. It starts with an overview of discriminant analysis, a stochastic representation of the discriminant function, classification rule and large dimension asymptotic approach. Fisher's linear discriminant analysis is then extended to discriminant analysis for repeated measurements with the help of the standard Growth Curve model and the Extended Growth Curve model. The chapter ends by presenting definitions and some results for the product of an inverse Wishart matrix and a normal vetor, $\mathbf{S}^{-1} \overline{\mathbf{X}}$, which is applied to portfolio theory, since it has similar structure to that of the linear discriminant function coefficients. Here the higher order moments of the estimated tangency portfolio weights, which include expressions for kurtosis and skewness, are given.
\end{abstract}

\subsection{Introduction}

There are many different multivariate statistical methods, for example factor analysis, cluster analysis, multivariate analysis of variance (MANOVA), generalized MANOVA, discriminant analysis, among others. Each method has its own type of analysis. Discriminant analysis is one of the method in multivariate statistics which can be described according to its tasks. The term "discriminant analysis" is commonly used interchangeably to represent two different goals. These goals of discriminant analysis are (i) description of group separation, and (ii) the classification of observations into groups. In the first goal, the discriminant functions that maximize the separation between the groups are used to identify the relative contribution of the $p$ variables that best allocate observations to the correct group. In the second goal, the classification function allocates a new observation 
into one of two or more given groups. The main classical methods in discriminant analysis are linear discriminant analysis (LDA) and quadratic discriminant analysis (QDA). The main assumption of the methods are; LDA assumes a common covariance matrix for all the groups, whereas QDA assumes that each class has its own covariance matrix. When $p$ is large QDA may be computational expensive.

In the 1930's multivariate statistics was blossoming and the first one in this time period to deal with discriminant analysis and classification analysis as we know it today was Fisher (1936). Before Fisher, developments of discriminant analysis and classification ignored correlations between the different variates in the feature vector, and considered only differences between groups based on sample moments, see for example Pearson (1916). Some years after these first attempts, Fisher published four papers on discriminant analysis, including Fisher (1938) in which he reviewed his 1936 work and related it to the contributions given by Hotelling (1931) and his famous $T^{2}$ statistic and by Mahalanobis (1936) on his $\Delta^{2}$ statistic as a distance measure.

Discriminant analysis can be applied in many areas, for example in applied psychology one may want to develop an efficient discriminant rule based on behaving, in medicine one may want to classify persons who are at high risk or low risk of contracting a specific disease, in credit scoring a bank may want to know if a new customer will be able to repay the loan or not based for example on age, income, debts or as a last example, in different industries one may want to determine when industry processes are in control or out of control.

\subsection{Stochastic representation of the discriminant function}

In the linear discriminant analysis the coefficients of the linear discriminant function are expressed as a product of the inverse covariance matrix and the difference of the mean vectors. Since we assume the covariance matrix to be positive definite, i.e., $\boldsymbol{\Sigma}>0$, the discriminant coefficients are given by

$$
\mathbf{a}=\boldsymbol{\Sigma}^{-1}\left(\boldsymbol{\mu}_{1}-\boldsymbol{\mu}_{2}\right)
$$

In practical situations the parameters $\boldsymbol{\mu}_{1}, \boldsymbol{\mu}_{2}$ and $\boldsymbol{\Sigma}$ are unknown and a can not be determined. Consequently, both $\boldsymbol{\mu}_{1}, \boldsymbol{\mu}_{2}$ and $\boldsymbol{\Sigma}$ need to be estimated. There are numerous estimation techniques for the mean vector (see Efron (2006)), the covariance matrix and for its inverse (see Fan et al. (2016), Bun et al. (2017)). Wald (1944) and Anderson (1951) suggested replacing the unknown parameters by the corresponding sample estimators. In the present thesis, we consider the classical unbiased sample estimators for $\boldsymbol{\mu}_{1}, \boldsymbol{\mu}_{2}$ and $\boldsymbol{\Sigma}$ 
which for two groups with $n_{1}$ and $n_{2}$ observations are expressed as

$$
\begin{aligned}
\overline{\mathbf{x}}_{i j} & =\frac{1}{n_{j}} \sum_{i=1}^{n_{j}} \mathbf{x}_{i j}=\frac{1}{n_{j}} \mathbf{X}^{(j)} \mathbf{1}_{n_{j}}, j=1,2, \\
\mathbf{S}_{p l} & =\frac{1}{n_{1}+n_{2}-2}\left[\left(n_{1}-1\right) \mathbf{S}^{(1)}+\left(n_{2}-1\right) \mathbf{S}^{(2)}\right],
\end{aligned}
$$

where

$$
\mathbf{S}^{(j)}=\frac{1}{n_{j}-1} \sum_{i=1}^{n_{j}}\left(\mathbf{x}_{i j}-\overline{\mathbf{x}}_{i}\right)\left(\mathbf{x}_{i j}-\overline{\mathbf{x}}_{i}\right)^{\prime} .
$$

Thus, replacing $\boldsymbol{\mu}_{1}, \boldsymbol{\mu}_{2}$ and $\boldsymbol{\Sigma}$ with $\overline{\mathbf{x}}_{1}, \overline{\mathbf{x}}_{2}$ and $\mathbf{S}_{p l}$ in (3.1), we obtain the sample discriminant function coefficients which are expressed as

$$
\widehat{\mathbf{a}}=\mathbf{S}_{p l}^{-1}\left(\overline{\mathbf{x}}_{1}-\overline{\mathbf{x}}_{2}\right) .
$$

In this thesis the focus is on the linear combination of the discriminant coefficients. In particular we are interested in

$$
\widehat{\theta}=\mathbf{l}^{\prime} \mathbf{S}_{p l}^{-1}\left(\overline{\mathbf{x}}_{1}-\overline{\mathbf{x}}_{2}\right),
$$

where 1 is a $p$-dimensional vector of constants. The linear combinations in (3.2) have several practical applications. For example, (i) it allows for a comparison of the sample coefficients in the linear discriminant function by deriving a corresponding statistical test, (ii) it can be used in the classification problem where given a new observation, one can decide to which predefined group an individual has to be assigned (discussed in detail in Paper A). By choosing $\mathbf{l}^{\prime}=(1,-1,0, \ldots, 0)$, difference between the first two discriminant coefficients can be analyzed based on a test. For example in Paper A Section 2.2, we have a one sided test for testing hypothesis for the equality of the first and the second coefficients in the population discriminant function. The hypothesis can be written as $H_{0}: \mathbf{l}^{\prime} \boldsymbol{\Sigma}^{-1}\left(\boldsymbol{\mu}_{1}-\boldsymbol{\mu}_{2}\right) \leq 0$ against $H_{1}: \mathbf{l}^{\prime} \boldsymbol{\Sigma}^{-1}\left(\boldsymbol{\mu}_{1}-\boldsymbol{\mu}_{2}\right)>0$. If $\mathbf{l}^{\prime}=(1,-1,0, \ldots, 0)$, rejection of the null hypothesis means: the first element of $\boldsymbol{\Sigma}^{-1}\left(\boldsymbol{\mu}_{1}-\boldsymbol{\mu}_{2}\right)$ is significantly larger than the second element of this vector. Then this means that the first variable appears to contribute more to the separation of the two groups.

In the present thesis the product $\mathbf{l}^{\prime} \mathbf{S}_{p l}^{-1}\left(\overline{\mathbf{x}}_{1}-\overline{\mathbf{x}}_{2}\right)$ is presented with the help of a stochastic representation using random variables from standard univariate distributions. This result is by itself very useful as it allows to generate values of $\mathbf{l}^{\prime} \mathbf{S}_{p l}^{-1}\left(\overline{\mathbf{x}}_{1}-\overline{\mathbf{x}}_{2}\right)$ by just generating the random variables from standard univariate distributions. The stochastic representation is also an important tool in analysing the distributional properties of $\mathbf{l}^{\prime} \mathbf{S}_{p l}^{-1}\left(\overline{\mathbf{x}}_{1}-\overline{\mathbf{x}}_{2}\right)$.

Some recent theoretical findings related to (3.2) have been obtained by Bodnar and Okhrin (2011) who derived the exact distribution of the product of the inverse sample 
covariance matrix and the sample mean vector. Kotsiuba and Mazur (2016) obtained the asymptotic distribution of functions of a Wishart matrix and a normal vetor as well as its approximate density based on an integral of the Gaussian function and a third order Taylor series expansion. In Paper A the product $\mathbf{l}^{\prime} \mathbf{S}_{p l}^{-1}\left(\overline{\mathbf{x}}_{1}-\overline{\mathbf{x}}_{2}\right)$ and its stochastic representation is used to obtain the asymptotic distribution under an assumption that the dimension $p$ of the parameter space increases together with the sample size $n$ to infinity such that the ratio $\frac{p}{n}$ converges to a positive constant $c \in(0,1)$. In Paper $\mathrm{D}$ the characterized form of the product in (3.2) has been applied to obtain formulas for higher order moments of the estimated tangency portfolio weights. The details of the stochastic representation of result (3.2) are given in Paper A. In the following we present a corollary of that paper.

\section{Corollary 3.1 (Paper A)}

Let $\mathbf{x}_{i j} \sim N\left(\boldsymbol{\mu}_{i}, \boldsymbol{\Sigma}\right), j=1, \ldots, n_{i}, i=1,2$. Let $\lambda=1 / n_{1}+1 / n_{2}, n_{1}+n_{2}-2>p$ and let $\mathbf{l}$ be as presented in (3.2). A stochastic representation of $\widehat{\theta}=\mathbf{l}^{\prime} \mathbf{S}_{p l}^{-1}\left(\overline{\mathbf{x}}_{1}-\overline{\mathbf{x}}_{2}\right)$ is given by

$$
\widehat{\theta} \stackrel{d}{=}\left(n_{1}+n_{2}-2\right) \xi^{-1}\left(\mathbf{l}^{\prime} \boldsymbol{\Sigma}^{-1}\left(\boldsymbol{\mu}_{1}-\boldsymbol{\mu}_{2}\right)+\sqrt{\left(\lambda+\frac{\lambda(p-1)}{n_{1}+n_{2}-p} u\right) \mathbf{l}^{\prime} \boldsymbol{\Sigma}^{-1} \mathbf{l} z_{0}}\right)
$$

where $\xi \sim \chi_{n_{1}+n_{2}-p-1}^{2}, z_{0} \sim N(0,1), u \sim \mathcal{F}\left(p-1, n_{1}+n_{2}-p,\left(\boldsymbol{\mu}_{1}-\boldsymbol{\mu}_{2}\right)^{\prime} \mathbf{R}_{\mathbf{l}}\left(\boldsymbol{\mu}_{1}-\right.\right.$ $\left.\boldsymbol{\mu}_{2}\right) / \lambda$ ) (non-central $\mathcal{F}$-distribution with $p-1$ and $n_{1}+n_{2}-p$ degrees offreedom and noncentrality parameter $\left.\left(\boldsymbol{\mu}_{1}-\boldsymbol{\mu}_{2}\right)^{\prime} \mathbf{R}_{\mathbf{l}}\left(\boldsymbol{\mu}_{1}-\boldsymbol{\mu}_{2}\right) / \lambda\right)$ with $\mathbf{R}_{\mathbf{l}}=\boldsymbol{\Sigma}^{-1}-\boldsymbol{\Sigma}^{-1} \mathbf{l l}^{\prime} \boldsymbol{\Sigma}^{-1} / \mathbf{l}^{\prime} \boldsymbol{\Sigma}^{-1} \mathbf{l}$; $\xi, z_{0}$ and $u$ are mutually independently distributed.

For the large dimensional case, the asymptotic distribution of $\mathbf{l}^{\prime} \mathbf{S}_{p l}^{-1}\left(\overline{\mathbf{x}}_{1}-\overline{\mathbf{x}}_{2}\right)$ is rewritten with the help of Corollary 3.1, under the assumption that the dimension $p$ increases together with the sample size $\left(n_{1}+n_{2}\right)$ and they all tend to infity in a way that $p /\left(n_{1}+n_{2}\right)$ converges to a known constant $c \in(0,1)$.

\subsection{Classification rule}

One goal of discriminant analysis is the allocation of observations to groups where the classification functions are employed to assign new observation to one of the groups. The technique is commonly applied in supervised classification problems. Supervised classification is a classification approach where an algorithm relies on labeled input data to estimate a function that predicts values of the outputs. Hastie et al. (2009) explores other supervised classification techniques which use an object characteristics to identify the class it belongs to, for example a new email is spam or non-spam or a patient should be diagnosed with a disease or not. These techniques include; $k$-nearest neighbours which is a classifier algorithm where the learning is based on how similar is a data vector from 
others. Another technique is Bayesian classification which is a method based on Bayes theorem. Moreover, a different supervised classification technique is neural networks, which estimates by an iterative algorithm and improves its performance after each iteration. A neural network contains layers of interconnected nodes: input layer, hidden layer and output layer. The number of layers varies from task to task, the more complex a task is, the more layers of neurons (as hidden layers) are used (see Aggarwal (2018), Ghatak (2019)). Moreover, one more important technique is support vector machine which finds the hyperplane that maximizes the gap between data points on the margins (so called "support vectors"). Some textbooks for example Johnson and Wichern (2007), Rencher and Christensen (2012), James et al. (2013) and Gutierrez (2015), have given detailed presentations of discriminant analysis and other supervised classification techniques.

In general the classification function should be interpreted as a separation of a set of all possible sample outcomes $\mathbb{R}^{p}$ into regions, say $R_{i}$, which are linked to groups $\pi_{i}$ in a way that for a new observation vector $\mathbf{x} \in R_{i}$ it is classified as a member of $\pi_{i}, i=1, \ldots, k$, for $k$ different groups. For the two group case a set in $\mathbb{R}^{p}$ is partitioned into regions $R_{1}$ and $R_{2}$ such that for $\mathbf{x} \in R_{1}$ an observation is classified into $\pi_{1}$. Let $L(\mathbf{x} ; \boldsymbol{\Theta})$ be Fisher's linear discriminant function for a random vector $\mathbf{x}$ and known parameter vector $\boldsymbol{\Theta}=\left(\boldsymbol{\mu}_{1}, \boldsymbol{\mu}_{2}, \boldsymbol{\Sigma}\right)$ given by

$$
L(\mathbf{x} ; \boldsymbol{\Theta})=\left(\boldsymbol{\mu}_{1}-\boldsymbol{\mu}_{2}\right)^{\prime} \boldsymbol{\Sigma}^{-1} \mathbf{x}-\frac{1}{2}\left(\boldsymbol{\mu}_{1}-\boldsymbol{\mu}_{2}\right)^{\prime} \boldsymbol{\Sigma}^{-1}\left(\boldsymbol{\mu}_{1}+\boldsymbol{\mu}_{2}\right),
$$

and if $L(\mathbf{x} ; \boldsymbol{\Theta})>0$ the observation vector $\mathbf{x}$, is classified to the first group $\pi_{1}$ and to the second group $\pi_{2}$ if $L(\mathbf{x} ; \boldsymbol{\Theta}) \leq 0$.

Let $f_{1}(\mathbf{x})$ represent the density function if $\mathbf{x}$ comes from $\pi_{1}$ and $f_{2}(\mathbf{x})$ be the density function if $\mathbf{x}$ comes from $\pi_{2}$, where $\pi_{1}$ and $\pi_{2}$ are multivariate normal distributed populations. The probability $\mathbb{P}(2 \mid 1)$ of classifying a new observation as $\pi_{2}$ when the observation comes from $\pi_{1}$ is given by

$$
\mathbb{P}(2 \mid 1)=\mathbb{P}\left(\mathbf{x} \in R_{2} \mid \mathbf{x} \in \pi_{1}\right)=\mathbb{P}\left(L(\mathbf{x} ; \boldsymbol{\Theta}) \leq 0 \mid \mathbf{x} \in \pi_{1}\right)=\int_{R_{2}} f_{1}(\mathbf{x}) d \mathbf{x},
$$

and $\mathbb{P}(1 \mid 2)$ is the probability to classify a new observation as $\pi_{1}$ when the observation belongs to $\pi_{2}$ is given by

$$
\mathbb{P}(1 \mid 2)=\mathbb{P}\left(\mathbf{x} \in R_{1} \mid \mathbf{x} \in \pi_{2}\right)=\mathbb{P}\left(L(\mathbf{x} ; \boldsymbol{\Theta})>0 \mid \mathbf{x} \in \pi_{2}\right)=\int_{R_{1}} f_{2}(\mathbf{x}) d \mathbf{x} .
$$

The probability of assigning the observation $\mathrm{x}$ into one group, when it actually comes from another group is called error rate or misclassification error.

In (3.3) the parameters $\boldsymbol{\mu}_{1}, \boldsymbol{\mu}_{2}, \boldsymbol{\Sigma}$ are unknown in practice, but as noted before they can be replaced by their sample estimators $\overline{\mathbf{x}}_{1}, \overline{\mathbf{x}}_{2}$, and $\mathbf{S}_{p l}$. It follows that the sample 
based classification function is given by

$$
\widehat{L}=\widehat{L}(\mathbf{x} ; \widehat{\boldsymbol{\Theta}})=\left(\overline{\mathbf{x}}_{1}-\overline{\mathbf{x}}_{2}\right)^{\prime} \mathbf{S}_{p l}^{-1}\left(\mathbf{x}-\frac{1}{2}\left(\overline{\mathbf{x}}_{1}+\overline{\mathbf{x}}_{2}\right)\right) \text {. }
$$

The distributional properties of the classification function $\widehat{L}$ can be analyzed and presented in a stochastic representation using random variables from standard univariate distributions (details are given in Paper A). The following theorem is presented.

\section{Theorem 3.1 (Paper A)}

Let $\lambda=1 / n_{1}+1 / n_{2}$. The stochastic representation of $\widehat{L}$ in (3.4) is given for $\mathbf{x} \in \pi_{i}, i=$ 1,2 ,

$$
\begin{aligned}
\widehat{L}(\mathbf{x} ; \widehat{\boldsymbol{\Theta}}) & \stackrel{d}{=} \frac{n_{1}+n_{2}-2}{\xi}\left((-1)^{i-1} \frac{\lambda n_{i}-2}{2 \lambda n_{i}}\left(\lambda \xi_{2}+\left(\Delta+\sqrt{\lambda} w_{0}\right)^{2}\right)\right. \\
& +\frac{(-1)^{i-1}}{\lambda n_{i}}\left(\Delta^{2}+\sqrt{\lambda} \Delta w_{0}\right)+\sqrt{\left(1+\frac{1}{n_{1}+n_{2}}+\frac{p-1}{n_{1}+n_{2}-p} u\right)} \\
& \left.\times \sqrt{\lambda \xi_{2}+\left(\Delta+\sqrt{\lambda} w_{0}\right)^{2}} z_{0}\right)
\end{aligned}
$$

where, $u \mid \xi_{1}, \xi_{2}, w_{0} \sim \mathcal{F}\left(p-1, n_{1}+n_{2}-p,\left(n_{1}+n_{2}\right)^{-1} \xi_{1}\right)$ with $\xi_{1} \mid \xi_{2}, w_{0} \sim \chi_{p-1, \delta_{\xi_{2}, w_{0}}^{2}}$ and $\delta_{\xi_{2}, w_{0}}^{2}=\frac{n_{1} n_{2}}{n_{i}^{2}} \frac{\Delta^{2} \xi_{2}}{\lambda \xi_{2}+\left(\Delta+\sqrt{\lambda} w_{0}\right)^{2}}$, both $z_{0}$ and $w_{0} \sim \mathcal{N}(0,1), \xi \sim \chi_{n_{1}+n_{2}-p-1}^{2}$, $\xi_{2}=\chi_{p-1}^{2} ; \xi, z_{0}$ are independent of $u, \xi_{1}, \xi_{2}, w_{0}$, where $\xi_{2}$ and $w_{0}$ are independent as well and $\Delta^{2}=\left(\boldsymbol{\mu}_{1}-\boldsymbol{\mu}_{2}\right)^{\prime} \boldsymbol{\Sigma}^{-1}\left(\boldsymbol{\mu}_{1}-\boldsymbol{\mu}_{2}\right)$.

To investigate the distributional properties of $\widehat{L}$ in a Monte Carlo study, it is sufficient to simulate only six random variables $\xi, \xi_{1}, \xi_{2}, z_{0}, w_{0}$, and $u$. Together with $\boldsymbol{\mu}_{1}, \boldsymbol{\mu}_{2}$, and $\boldsymbol{\Sigma}$ via the quadratic form $\Delta$ in the representation, the distribution of $\widehat{L}$ can be generated. The result in Theorem 3.1 does not hold when $p$ is comparable to $n$. In Paper A analytical expression for the asymptotic distribution of this result under the large-dimensional asymptotics is presented.

\subsection{Large dimension asymptotic approach}

The advancement of science and technology has brought new challenges of handling highdimensional data sets, where the dimension of the parameter space can be in thousands. For example in microarray data classification, face recognition, text mining, often the sample size is small and the size of the parameter space is large. Also in finance, particularly in portfolio theory, the number of financial stocks (features or parameters) are sometimes comparable to the number of observations in the portfolio. In such situations, 
the ways we arrange the high-dimensional scenarios in an asymptotic set-up is paramount. In a high-dimensional framework the size of the sample space $(n)$ is smaller than the size of the parameter space $(p)$, whereas in a large-dimensional framework the dimension of the parameter space is still smaller than the size of the sample space but of the same order. Thus, we can assume the following settings;

(i) $p>n$ and $p / n$ converges to a positive constant $c>1, p$ gets to infinity faster than $n$;

(ii) $p \approx n$ and $p / n$ converges to positive constant $c \in(0,1), p$ and $n$ grow with the same speed.

One of the first statistical methods which was modified to handle high-dimensional data was discriminant analysis. Early contributions on the curse of dimensionality was by the late A. N. Kolmogorov and his colleagues at his statistical methods laboratory at Moscow University.

The asymptotic approach called "double asymptotic approach" for analyzing the case when the sample size $n$ and the dimension $p$, are growing simultaneously at a constant rate, was first given by Raudys (1967), and is now widely applied in investigating the error (difference between the error of the training data and the one of the test data) of the statistical classification algorithms such as artificial neural networks and pattern recognition algorithms. The approach allows one to obtain accurate estimates when the sample size is comparable with the number of features, (Raudys (2012)). Deev (1970) was inspired by the study of Raudys (1967) and formalized the double asymptotic approach in a strictly mathematical way, by proposing a growing dimension asymptotic approach, where both the dimension $p$ and sample size $n$ get large such that $p / n$ converges to a positive constant, as $p \rightarrow \infty, n \rightarrow \infty$. He argued that the standard asymptotics, that is, $p$ assumed fixed and $n$ goes to infinity, is too restricted for the analysis of discrimination performance. Again Raudys (1972) published a paper with results that distinguish complexity of classification rules for the standard Fisher linear discriminant function, the Euclidean distance classifier, the diagonal classifier and regularized linear discriminant analysis, according to the structure of the covariance matrices. Studies done by Raudys, Deev and other early Russian researchers in discriminant analysis appear in an overview (Raudys and Young (2004)) of important contributions by Soviet researchers to topics in the discriminant analysis literature concerning the small training sample size problem.

Furthermore, Friedman (1989) formulated the problem of regularization of the covariance matrix in linear discriminant analysis. He employed a ridge type approach that is based on adding of a diagonal matrix to the sample covariance matrix. Later Raudys and Jain (1991) studied the effects of sample size such as $n_{1} \neq n_{2}$ on feature selection and error estimation for different classification methods. Fujikoshi and Seo (1998) provided an 
asymptotic approximation of the linear discriminant function in large dimension by considering the case of unequal sample sizes and compared the results with the asymptotic approximation by Wyman et al. (1990). For the samples of non-equal sizes, Fujikoshi and Seo (1998) pointed out that the large-dimensional approximation is extremely accurate. Other scholars have written about large dimension asymptotics, e.g., Pavlenko and von Rosen (2001) obtained the asymptotic expression for the error probabilities and consistent approximation of the discriminant function. They proposed different types of relations between the dimensionality and the size of the training sample.

Also in recent years there are studies in linear discriminant analysis which evaluate the misclassification errors. For example Hyodo et al. (2015) and Watanabe et al. (2015) expanded the expression for the asymptotic approximation for the misclassification errors stochastically using Taylor series expansion. The paper by Fujikoshi (2000) and Chapter 16 of Fujikoshi et al. (2010) have many details on the Taylor series expansion of the asymptotic misclassification expression and their possible errors of approximations. In Paper B and C we have used an approach of substituting the expectations of the expression for the approximations of misclassification errors by following ideas given in Fujikoshi (2000) and Shutoh et al. (2011). In Paper A Section 3.1, we provide an expression for the asymptotic distribution of the linear classification function under the assumption that the dimension increases together with the sample size such that $p / n$ converges to $c \in(0,1)$, as $n \rightarrow \infty$.

\subsection{Classification of growth curves}

In Section 3.4 the linear discriminant function and all results rely on data in which several variables are collected at a single time point. In this section we present classification of growth curves using repeated measurements. The classification function is modified and applied to data collected for the same individual at several time points.

Repeated measures data are common in many applications for example in pharmacy, medical research studies, agricultural studies, business studies and environmental research. The Growth Curve model is a typical model for such data. It is for this type of data, classification of growth curves will be considered in this section as well as in $\mathrm{Pa}$ per B and C. This section introduces basics for the development of the linear discriminant function with the means following the Growth Curve model and the Extended Growth Curve model.

Linear discriminant analysis with repeated measurements has been been studied for a long time. Burnaby (1966) was one of the earliest scholars to consider discriminant analysis for measurements taken on the same subject at different time points. The focus was to generalize procedures of discrimination as well as to propose a generalized distance 
between the populations of repeated measurements. Burnaby's approach did not rely on the Growth Curve model introduced by Potthoff and Roy (1964). Later Lee (1977) used a Bayesian approach to classify observation following the Growth Curve model. The study by Lee (1977) was generalized by Nagel (1979). Again Lee (1982) developed classification procedures for growth curves using Bayesian and non-Bayesian methods. The study by Lee (1982) considered two different covariance structures, an arbitrary positive definite covariance matrix and Rao's simple covariance structure (Rao (1967)).

Furthermore, Mentz and Kshirsagar (2005) considered classification of growth curves with means following the Potthoff and Roy (1964) Growth Curve model and computed the misclassification errors by a Monte Carlo simulation study. The misclassification errors computed using formulated classification function were compared based on arbitrary matrix and structured covariancematrices (compound symmetry covariance and Rao's simple covariance structure). Classification of multivariate repeated measures data has also been considered by Roy and Khattree (2005a,b, 2007). They studied classification in small samples by assuming a Kronecker product structure on the covariance matrix. They assumed equicorrelated or compound symmetry correlation structure on the repeated measures in their $2005 \mathrm{~b}$ paper, and an autoregressive model of order one, $A R(1)$, for the repeated measurements in their other two papers.

Our study of obtaining misclassification errors in Paper B and C follow the approach given in Fujikoshi (2000). The approximations of misclassification errors we propose is useful for repeated measures data.

Usually in medical research several decisions or identification of problems are based on examinations which involve measurements taken over time. For instance, we may be interested in identifying whether a tumor is a benign (non cancerous) tumor or a malignant (cancerous) tumor based on measurements of several examinations. In this kind of situation, the challenge is to construct a decision rule based on these repeated measurements that can be used to discriminate between healthy subjects (non cancerous) and patients (cancerous).

Suppose that there are two groups, $\pi_{1}$ and $\pi_{2}$, that is

$$
\pi_{i}: \mathbf{x}^{(i)}=\mathbf{A b}_{i}+\mathbf{e}, \mathbf{e} \sim N_{p}(\mathbf{0}, \boldsymbol{\Sigma}), \quad i=1,2 .
$$

The goal is to allocate a new observation $\mathbf{x}$ of $p$ repeated measurements into one of these groups. Following the likelihood based decision rule in Paper B we have the classification function

$$
L\left(\mathbf{x} ; \mathbf{b}_{1}, \mathbf{b}_{2}, \mathbf{\Sigma}\right)=\left(\mathbf{b}_{1}-\mathbf{b}_{2}\right)^{\prime} \mathbf{A}^{\prime} \boldsymbol{\Sigma}^{-1} \mathbf{A}\left(\mathbf{x}-\frac{1}{2}\left(\mathbf{b}_{1}+\mathbf{b}_{2}\right)\right) .
$$

The discrimination rule based on (3.5) is defined by

allocate $\mathbf{x}$ to $\pi_{1}$ if $L\left(\mathbf{x} ; \mathbf{b}_{1}, \mathbf{b}_{2}, \boldsymbol{\Sigma}\right)>0$ and to $\pi_{2}$ otherwise. 
Unlike the Fisher classification rule (3.3), the discriminant function in (3.5) has group means following the Growth Curve model. As a result the function in (3.5), can classify a new observation $\mathbf{x}$ of $p$ repeated measurements into one of the predefined groups. $L\left(\mathbf{x} ; \mathbf{b}_{1}, \mathbf{b}_{2}, \mathbf{\Sigma}\right)$ solely depends on the population parameters $\mathbf{b}_{1}, \mathbf{b}_{2}$ and $\boldsymbol{\Sigma}$, which are unknown in practice and must be estimated from training data. Hence, the classification rule based on the sample estimators is given as $L\left(\mathbf{x} ; \widehat{\mathbf{b}}_{1}, \widehat{\mathbf{b}}_{2}, \widehat{\mathbf{\Sigma}}\right)$.

In the following we present an example that uses the benign and malignant tumor data set, given in Section 2.3.

\section{Example 3.1: Example 2.2 continued}

Assume that we have a new patient with a tumor. We decide to perform 10 repeated measurements of the size of the tumor and obtain

$$
\mathbf{x}=(0.13,0.24,1.82,2.26,2.41,1.21,-0.33,-0.14,0.30,-0.21)
$$

Given the training data set in Table 2.1, a tumor $\mathbf{x}$ may be classified as being a benign or malignant tumor.
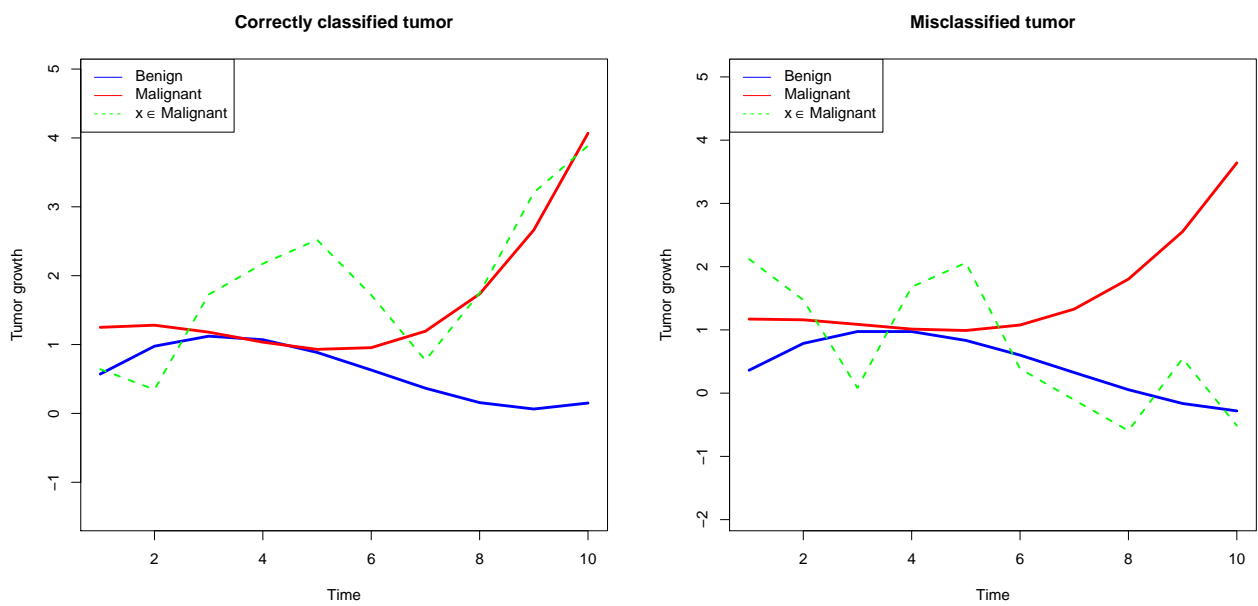

Figure 3.1: Correctly classifed and misclassified new observation (tumors) for $\pi_{1}$ and $\pi_{2}$. Red lines represents estimated growth profiles for the malignant tumor and blue lines the estimated growth profiles for the benign tumor.

In Figure 3.1 (left), $L(\mathbf{x})=80.7>0$, that is, the tumor is classified as malignant, which is correct, whereas in Figure 3.1 (right), $L(\mathbf{x})=-36.7<0$, that is, the tumor is classified to belong to the benign group, but it actually belongs to the malignant group. 
It is difficult to obtain an expression of the exact probability of misclassifications (Fujikoshi et al. (2010)). Alternatively, misclassification errors can be evaluated using approximations. In Paper B, the results for approximations of misclassifcation errors are given. If $\boldsymbol{\Sigma}$ is known and observation $\mathrm{x}$ of $p$ repeated measurements is assumed to come from $\pi_{1}$, then $L$ can be expressed in terms of location $U$ and scale $V$ mixture of the standard normal distribution;

$$
L=V^{1 / 2} Z-U
$$

where

$$
\begin{aligned}
V & =\left(\widehat{\mathbf{b}}_{1}-\widehat{\mathbf{b}}_{2}\right)^{\prime} \mathbf{A}^{\prime} \boldsymbol{\Sigma}^{-1} \mathbf{A}\left(\widehat{\mathbf{b}}_{1}-\widehat{\mathbf{b}}_{2}\right), \\
Z & =V^{-1 / 2}\left(\widehat{\mathbf{b}}_{1}-\widehat{\mathbf{b}}_{2}\right)^{\prime} \mathbf{A}^{\prime} \boldsymbol{\Sigma}^{-1}\left(\mathbf{x}-\mathbf{A} \mathbf{b}_{1}\right), \\
U & =\left(\widehat{\mathbf{b}}_{1}-\widehat{\mathbf{b}}_{2}\right)^{\prime} \mathbf{A}^{\prime} \boldsymbol{\Sigma}^{-1} \mathbf{A}\left(\widehat{\mathbf{b}}_{1}-\mathbf{b}_{1}\right)-\frac{1}{2} V .
\end{aligned}
$$

Moreover, conditioned on $\widehat{\mathbf{b}}_{1}$ and $\widehat{\mathbf{b}}_{2}, Z$ is independent of $U$ and $V$ and is distributed as $N(0,1)$. The probability of misclassification where $\mathbf{x}$ is assigned to $\pi_{2}$ when it actually belongs to $\pi_{1}$ can be expressed using (3.6) as

$$
\mathbb{P}(2 \mid 1)=e(2 \mid 1)=\operatorname{Pr}\left(L \leq 0 \mid \mathbf{x} \in \pi_{1}, \widehat{\mathbf{b}}_{1}, \widehat{\mathbf{b}}_{2}, \boldsymbol{\Sigma}\right)=\mathbb{E}_{(U, V)}\left[\Phi\left(V^{-1 / 2} U\right)\right],
$$

where $\Phi(\cdot)$ is the distribution function of the standard normal distribution. Fujikoshi et al. (2010), Hyodo et al. (2015) and Watanabe et al. (2015) have been expanding the expression given in (3.7) using a Taylor series expansion, to evaluate the misclassification errors. Following the ideas of Fujikoshi (2000), an approximation of (3.7), the misclassification error, is given in Paper B as

$$
e(2 \mid 1) \simeq \Phi\left(\{\mathbb{E}[V]\}^{-1 / 2} \mathbb{E}[U]\right),
$$

where the expectations $\mathbb{E}[V]$ and $\mathbb{E}[U]$ are given by

$$
\begin{aligned}
& \mathbb{E}[V]=\Delta^{2}+\frac{n_{1}+n_{2}}{n_{1} n_{2}} q, \\
& \mathbb{E}[U]=-\frac{1}{2}\left(\Delta^{2}+\frac{n_{1}-n_{2}}{n_{1} n_{2}} q\right),
\end{aligned}
$$

and

$$
\Delta^{2}=\left(\mathbf{b}_{1}-\mathbf{b}_{2}\right)^{\prime} \mathbf{A}^{\prime} \boldsymbol{\Sigma}^{-1} \mathbf{A}\left(\mathbf{b}_{1}-\mathbf{b}_{2}\right),
$$

is identical to the squared Mahalanobis distance. Inserting (3.9) and (3.10) in (3.8) leads to

$$
e(2 \mid 1) \simeq \Phi(\alpha)
$$


where

$$
\alpha=-\frac{1}{2}\left(\frac{\Delta^{2}+\frac{n_{1}-n_{2}}{n_{1} n_{2}} q}{\sqrt{\Delta^{2}+\frac{n_{1}+n_{2}}{n_{1} n_{2}} q}}\right) .
$$

If $n_{1}, n_{2}$ tend to infinity, the approximation in (3.11) equals $e(2 \mid 1) \simeq \Phi\left(-\frac{1}{2} \Delta\right)$, since $\lim _{n_{1}, n_{2} \rightarrow \infty} \mathbb{E}[V]=\Delta^{2}$ and $\lim _{n_{1}, n_{2} \rightarrow \infty} \mathbb{E}[U]=-\frac{1}{2} \Delta^{2}$. Suppose that $n_{1}>n_{2}$, the approximation will be $e(2 \mid 1) \simeq \Phi\left(-\frac{1}{2}\left(\Delta^{2}+q\right)\left(\Delta^{2}+q\right)^{-\frac{1}{2}}\right)$. However, an attempt to compare the performance of the approximation in the case $n_{1} \neq n_{2}$ is not considered in this thesis. An estimator of $\Delta^{2}$ may be obtained by simply replacing the unknown parameters $\mathbf{b}_{1}$ and $\mathbf{b}_{2}$ with appropriate estimators $\widehat{\mathbf{b}}_{1}$ and $\widehat{\mathbf{b}}_{2}$. The proposed approximation which results in (3.11) can be extended for the case when $\boldsymbol{\Sigma}$ is unknown. If $\boldsymbol{\Sigma}$ is unknown and the dimension $p$ is allowed to increase with the sample size $n$, then the statistic $\Delta^{2}$ becomes instable and can result in an increase of the misclassification errors.

The Extended Growth Curve model was introduced in Chapter 2. Following the likelihood based decision rule given in Paper B we obtain a generalized classification function:

$$
L\left(\mathbf{x} ; \mathbf{b}_{1}, \mathbf{b}_{2}, \boldsymbol{\Sigma}\right)=\left(\mathbf{A}_{1} \mathbf{b}_{1}-\mathbf{A}_{2} \mathbf{b}_{2}\right)^{\prime} \boldsymbol{\Sigma}^{-1}\left(\mathbf{x}-\frac{1}{2}\left(\mathbf{A}_{1} \mathbf{b}_{1}+\mathbf{A}_{2} \mathbf{b}_{2}\right)\right) .
$$

The new observation $\mathrm{x}$ consisting of $p$ repeated measurements is assigned to $\pi_{1}$ if $L\left(\mathbf{x} ; \mathbf{b}_{1}, \mathbf{b}_{2}, \mathbf{\Sigma}\right)>0$ and to $\pi_{2}$ otherwise.

It is difficult to find an expression that can evaluate the exact probability of misclassification. As a result many authors resort on approximations. In Paper C, an approximations for the probability of misclassification is proposed in the same way as in Paper B, when using the standard growth curve structure given in Potthoff and Roy (1964). The approximation for the probability of misclassification for large $n$ and $p$ is

$$
e(2 \mid 1) \simeq \Phi\left(\{\mathbb{E}[V]\}^{-1 / 2} \mathbb{E}[U]\right),
$$

where

$$
\begin{aligned}
V & =\left(\mathbf{A}_{1} \widehat{\mathbf{b}}_{1}-\mathbf{A}_{2} \widehat{\mathbf{b}}_{2}\right)^{\prime} \boldsymbol{\Sigma}^{-1}\left(\mathbf{A}_{1} \widehat{\mathbf{b}}_{1}-\mathbf{A}_{2} \widehat{\mathbf{b}}_{2}\right), \\
U & =\left(\mathbf{A}_{1} \widehat{\mathbf{b}}_{1}-\mathbf{A}_{2} \widehat{\mathbf{b}}_{2}\right)^{\prime} \boldsymbol{\Sigma}^{-1} \mathbf{A}_{1}\left(\widehat{\mathbf{b}}_{1}-\mathbf{b}_{1}\right)-\frac{1}{2} V .
\end{aligned}
$$

In Paper $\mathrm{C}$ we derive $\mathbb{E}[V]$ and $\mathbb{E}[U]$;

$$
\begin{aligned}
& \mathbb{E}[V]=\Delta^{2}+\frac{n_{1}+n_{2}}{n_{1} n_{2}} q_{1}, \\
& \mathbb{E}[U]=-\frac{1}{2}\left(\Delta^{2}+\frac{n_{1}-n_{2}}{n_{1} n_{2}} q_{1}\right),
\end{aligned}
$$

where

$$
\Delta^{2}=\left(\mathbf{A}_{1} \mathbf{b}_{1}-\mathbf{A}_{2} \mathbf{b}_{2}\right)^{\prime} \boldsymbol{\Sigma}^{-1}\left(\mathbf{A}_{1} \mathbf{b}_{1}-\mathbf{A}_{2} \mathbf{b}_{2}\right), \quad \text { and } \quad q_{1}=\operatorname{rank}\left(\mathbf{A}_{1}\right) .
$$


Inserting (3.13) and (3.14) in (3.12) we get

$$
e(2 \mid 1) \simeq \Phi(\widetilde{\alpha})
$$

where

$$
\widetilde{\alpha}=-\frac{1}{2}\left(\frac{\Delta^{2}+\frac{n_{1}-n_{2}}{n_{1} n_{2}} q_{1}}{\sqrt{\Delta^{2}+\frac{n_{1}+n_{2}}{n_{1} n_{2}} q_{1}}}\right) .
$$

If $n_{1}, n_{2}$ are allowed to increase to infinity, with $n_{1} / n_{2}$ tending to a positive constant, then the approximation in (3.12) becomes $e(2 \mid 1) \simeq \Phi\left(-\frac{1}{2} \Delta\right)$, since $\lim _{n_{1}, n_{2} \rightarrow \infty} \mathbb{E}[V]=\Delta^{2}$ and similarly $\lim _{n_{1}, n_{2} \rightarrow \infty} \mathbb{E}[U]=-\frac{1}{2} \Delta^{2}$. If $n_{1}>n_{2}$, the approximation becomes $e(2 \mid 1) \simeq \Phi\left(-\frac{1}{2}\left(\Delta^{2}+q_{1}\right)\left(\Delta^{2}+q_{1}\right)^{-\frac{1}{2}}\right)$. As before, $\Delta^{2}$ is the squared Mahalanobis distance. Large $\Delta^{2}$ results in small misclassification errors.

\subsection{Applications to Portfolio theory}

In Section 3.2 the product of the inverse Wishart matrix and the normal distributions was used when linear discriminant function coefficients were studied. In this section the product of the inverse Wishart matrix and the normal distribution find its applications in portfolio theory, introduced by Markowitz (1952). This implies that the theory introduced in Section 3.2 can be directly applied to the components of $\mathbf{S}^{-1}\left(\overline{\mathbf{x}}_{1}-\overline{\mathbf{x}}_{2}\right)$ by replacing $\overline{\mathbf{x}}_{1}-\overline{\mathbf{x}}_{2}$ with a random normal vector $\mathbf{x}$. In portfolio theory, the vector of optimal portfolio weights is proportional to the product of inverse the Wishart matrix and a normal vector. In this section we give definitions and some results of this product. In Paper D of the thesis the product has been applied and provided analytical results for higher order moments of the estimated tangency portfolio weights, which also include expressions for skewness and kurtosis.

The mean-variance portfolio theory for asset allocation was first published by Markowitz (1952) in his seminal paper. The theory plays a central role in allocating the investments among different assets. This section aims at defining and giving basic understanding of porfolio theory. It focuses on properties of tangency portfolio (TP) weights. Let $\mathbf{x}_{t}=\left(x_{11}, \ldots, x_{p t}\right)^{\prime}$ be a $k$-dimensional column vector of log-returns, covering $p$ assets at time $t=1, \ldots, n$. Denote the vector of weights by $\mathbf{w}=\left(w_{1}, \ldots, w_{p}\right)^{\prime}$, where $w_{i}$ is the weight of the $i$-th asset in the portfolio. The weights are proportional to the value of the assets within the portfolio. In the mean-variance portfolio optimization, the investor allocates the wealth among assets by maximizing the expected return $\mathbb{E}\left[R_{p}\right]$ subject to given level of the risk $\operatorname{Var}\left(R_{p}\right)$ or by minimizing the risk for a given level of expected return. Usually the risk is measured by the variance of the portfolio return $\operatorname{Var}\left(R_{p}\right)$. The investor determines the proportion of wealth allocated to each asset by maximizing 
the expected quadratic utility $\mathbb{E}\left[R_{p}\right]-\frac{\alpha}{2} \operatorname{Var}\left(R_{p}\right)$, where $\mathbb{E}\left[R_{p}\right]$ and $\operatorname{Var}\left(R_{p}\right)$ are respectively the portfolio return and variance, $\alpha$ is the risk aversion coefficient (investor's attitude or feeling toward risk). In case there is portfolio allocation without risk free asset, the expected return on the portfolio is given by $\mathbb{E}\left[R_{p}\right]=\mathbf{w}^{\prime} \boldsymbol{\mu}$ and its variance is equal to $\operatorname{Var}\left(R_{p}\right)=\mathbf{w}^{\prime} \boldsymbol{\Sigma} \mathbf{w}$. Then the set of optimal portfolios that offer the highest expected return for a given level of risk or the lowest risk for a defined level of expected return (efficient frontier) is fully determined by maximizing the expected quadratic utility, that is

$$
\operatorname{maximize} \mathbf{w}^{\prime} \boldsymbol{\mu}-\frac{\alpha}{2} \mathbf{w}^{\prime} \boldsymbol{\Sigma} \mathbf{w}, \quad \text { subject to } \mathbf{w}^{\prime} \mathbf{1}=1 \text {. }
$$

By $\mathbf{w}^{\prime} \mathbf{1}=1$, means that we require the investor to be fully invested. Markowitz (1952) showed that by solving the problem in (3.16) for different values of the risk aversion coefficient $\alpha>0$, a set of efficient portfolios is obtained. In the presence of risk free asset, the expected return on the portfolio is given by $\mathbb{E}\left[R_{p}\right]=\mathbf{w}^{\prime}\left(\boldsymbol{\mu}-r_{f} \mathbf{1}_{p}\right)+r_{f}$, where $r_{f}$ is the rate of return on the risk free asset. The portfolio weights are obtained by maximizing the following quadratic utility function with respect to $w$ :

$$
\operatorname{maximize} \mathbf{w}^{\prime}\left(\boldsymbol{\mu}-r_{f} \mathbf{1}_{p}\right)+r_{f}-\frac{\alpha}{2} \mathbf{w}^{\prime} \mathbf{\Sigma} \mathbf{w} \quad \text { subject to } \mathbf{w}^{\prime} \mathbf{1}=1,
$$

By application of Lagrange multiplier, the solution of the maximization problem (3.17) is given by

$$
\mathbf{w}_{T P}=\alpha^{-1} \boldsymbol{\Sigma}^{-1}\left(\boldsymbol{\mu}-r_{f} \mathbf{1}_{p}\right), w_{r}=1-\mathbf{1}^{\prime} \mathbf{w}
$$

where $w_{r}$ is the fraction of investor's wealth invested into the risk free asset (see Figure 3.2). Suppose we have a portfolio where the whole wealth is invested into risky assets, then its weights are given by

$$
\mathbf{w}_{T P *}=\frac{\boldsymbol{\Sigma}^{-1}\left(\boldsymbol{\mu}-r_{f} \mathbf{1}_{p}\right)}{\mathbf{1}^{\prime} \boldsymbol{\Sigma}^{-1}\left(\boldsymbol{\mu}-r_{f} \mathbf{1}_{p}\right)}, w_{r}=0,
$$

and it is assumed that $\mathbf{1}^{\prime} \boldsymbol{\Sigma}^{-1}\left(\boldsymbol{\mu}-r_{f} \mathbf{1}_{p}\right) \neq 0$. This portfolio is also known as a tangency porfolio weights (see, e.g., Ingersoll (1987), Britten-Jones (1999), Bodnar and Schmid (2009)). When $r_{f}=0$ in (3.19) it means that the whole wealth is invested into the risky asset (at the tangent point) only. This case leads to a portfolio weights with highest Sharpe ratio (an important criteria in asset allocation). A detailed discussion of the tangency portfolio is given for example in Ingersoll (1987).

However, in practice, $\boldsymbol{\mu}$ and $\boldsymbol{\Sigma}$ are unknown quantities and must be estimated from data. Then, we consider a random sample of asset returns $\mathbf{x}_{1}, \ldots, \mathbf{x}_{n}$ which are assumed to be independent and multivariate normally distributed with mean $\boldsymbol{\mu}$ and covariance matrix $\boldsymbol{\Sigma}$. Using the random samples of asset returns, we estimate the unknown parameters 
$\boldsymbol{\mu}$ and $\boldsymbol{\Sigma}$ by their corresponding sample estimators given by

$$
\overline{\mathbf{x}}=\mathbf{X} \mathbf{1}_{n}\left(\mathbf{1}_{n}^{\prime} \mathbf{1}_{n}\right)^{-1}, \mathbf{S}=\frac{1}{n-1} \mathbf{X}\left(\mathbf{I}-\mathbf{1}_{n}\left(\mathbf{1}_{n}^{\prime} \mathbf{1}_{n}\right)^{\prime} \mathbf{1}_{n}\right) \mathbf{X}^{\prime}, \mathbf{X}=\left(\mathbf{x}_{1}, \ldots, \mathbf{x}_{n}\right) .
$$

Replacing $\boldsymbol{\mu}$ and $\boldsymbol{\Sigma}$ with their respetive estimators $\overline{\mathbf{x}}$ and $\mathbf{S}$ in (3.18) and (3.19), we get the following estimated porfolio weights

$$
\begin{aligned}
\widehat{\mathbf{w}}_{T P} & =\frac{1}{\alpha} \mathbf{S}^{-1}\left(\overline{\mathbf{x}}-r_{f} \mathbf{1}_{p}\right) \\
\widehat{\mathbf{w}}_{T *} & =\frac{\mathbf{S}^{-1}\left(\overline{\mathbf{x}}-r_{f} \mathbf{1}_{p}\right)}{\mathbf{1}^{\prime} \mathbf{S}^{-1}\left(\overline{\mathbf{x}}-r_{f} \mathbf{1}_{p}\right)}, \quad w_{r}=0 .
\end{aligned}
$$

Fama (1976) pointed out that the normal assumption has been found to be appropriate for describing the distribution of monthly return data. Moreover, Tu and Zhou (2004) discussed that the normal assumption works well for the mean-variance investor. Despite the fact that, the fat tails of asset return distribution influences the estimators of the portfolio weights, expected return and variance, the impact is not large. They further pointed out that the investor who ignores the heavy tails has relatively small losses. Fama and French (2012) mentioned that the distributions of daily and monthly stock returns are rather symmetric about their means, but the tails are fatter (that is, there are more outliers) than would be expected with the normal distributions. In Paper D the skewness and kurtosis have been studied to measure the deviation from the normal distribution. Moreover, the moments of the estimated tangency portfolio weights have been computed in order to study their behaviour when the assumption of normality is violated.

Considering the sample estimators of $\boldsymbol{\mu}$ and $\boldsymbol{\Sigma}$ that are given in (3.20) and assuming normality, we have that

$$
\overline{\mathbf{x}} \sim N_{p}\left(\boldsymbol{\mu}, \frac{1}{n} \boldsymbol{\Sigma}\right) \text { and }(n-1) \mathbf{S} \sim W_{p}(n-1, \boldsymbol{\Sigma}),
$$

and, $\overline{\mathbf{x}}$ and $\mathbf{S}$ are independently distributed.

We can observe that the sample estimator $\widehat{\mathbf{w}}_{T P}$ of the tangency portfolio weights $\mathbf{w}_{T P}$ given in (3.21) is expressed as the product of an inverse Wishart matrix and a normal random vector. Components in the portfolio theory can be described in expressions which depend on $\mathbf{S}^{-1} \mathbf{x}$, where $\mathbf{S}$ is a Wishart matrix and $\mathbf{x}$ is a Gaussian vector, which is independent of $\mathbf{S}$. Suppose an arbitrary $\mathbf{L}: k \times p$ matrix of constants is considered such that $\mathbf{L S}^{-1} \mathbf{x}$, then one can explore several linear combinations of the elements of the random vector $\mathbf{S}^{-1} \mathbf{x}$. In this thesis a special attention is taken to the case when $\mathbf{L}$ is a vector. The distribution of the function possesses practical applications, for example, it allows one to develop a test statistic that can be used to compare the population coefficients, so that more insight into the structure of the elements of $\mathbf{S}^{-1} \mathbf{x}$ can be explored. For example if we choose $\mathbf{l}=(1,0,0, \ldots, 0)^{\prime}$ then an investor can know the behaviour of 


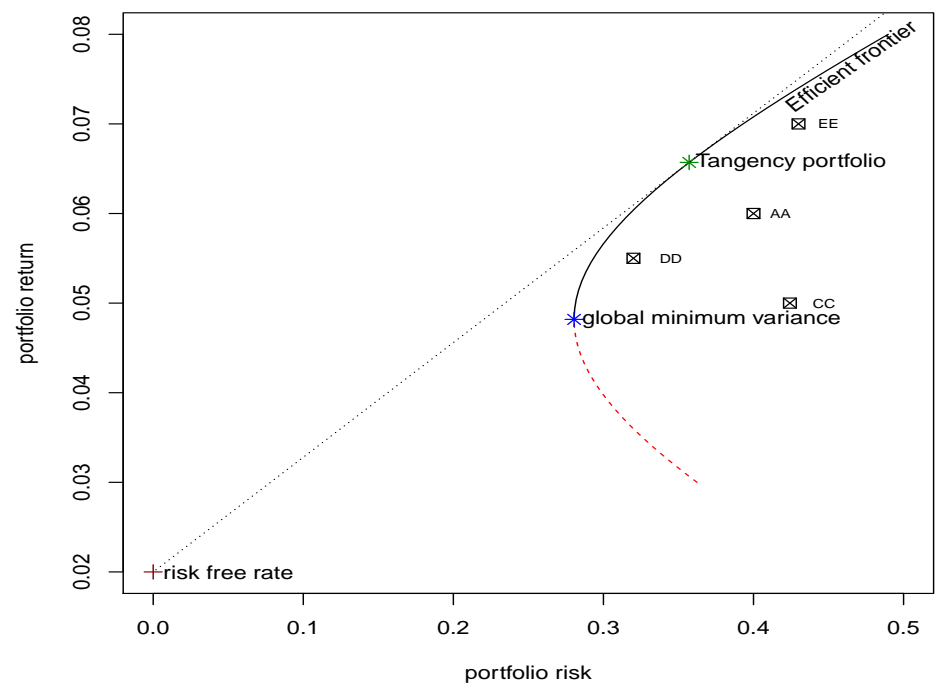

Figure 3.2: Graphical illustration of the tangency portfolio in the presence of the risk-free asset and efficient frontier. $A A, C C, D D$ and $E E$ are the portfolios.

the tangency portfolio weight of the first asset. Likewise, if $\mathbf{l}=(1,1,0, \ldots, 0)^{\prime}$, then we can analyse the behaviour of the sum of the first two assets in the portfolio. This implies that the theoretical results given in Theorem 3.1 (Paper A) Section 3.1, can be applied to the components of $\widehat{\mathbf{w}}_{T P}$ given in (3.18) by replacing $\overline{\mathbf{x}}_{1}-\overline{\mathbf{x}}_{2}$ with $\overline{\mathbf{x}}$. In the second part of the present thesis, the distribution of the coefficients has been used to provide the analytical results for higher order moments of the estimated tangency portfolio weights, which also include the expressions for skewness and kurtosis.

Below is the distribution of $\mathbf{l}^{\prime} \mathbf{S}^{-1} \mathbf{x}$ which is characterized in terms of random variables from standard distributions. This results is presented in detail in Paper D, and it plays an important role in the derivations of higher order moments of $\mathbf{l}^{\prime} \mathbf{S}^{-1} \mathbf{x}$.

\section{Lemma 3.1 (Paper D)}

Let $\mathbf{S} \sim W_{p}(n, \boldsymbol{\Sigma}), n>p$ and $\mathbf{x} \sim N_{p}(\boldsymbol{\mu}, \lambda \boldsymbol{\Sigma})$ with $\lambda>0$ and positive definite $\mathbf{\Sigma}$. Furthermore, let $\mathbf{S}$ and $\mathbf{x}$ be independent and $\mathbf{l}$ be a p-dimensional vector of constants. Then the stochastic representation of $\mathbf{1}^{\prime} \mathbf{S}^{-1} \mathbf{x}$ is given by

$$
\mathbf{l}^{\prime} \mathbf{S}^{-1} \mathbf{x} \stackrel{d}{=} \frac{1}{u_{1}}\left(\mathbf{l}^{\prime} \boldsymbol{\Sigma}^{-1} \boldsymbol{\mu}+\sqrt{\left.\left(\lambda+\frac{\lambda(p-1)}{n-p+2} u_{3}\right) \mathbf{l}^{\prime} \boldsymbol{\Sigma}^{-1} \mathbf{l} u_{2}\right)},\right.
$$

where $u_{1} \sim \chi_{n-p+1}^{2}, u_{2} \sim \mathcal{N}(0,1)$ and $u_{3} \sim \mathcal{F}(p-1, n-p+2, s)$ with $s=\boldsymbol{\mu}^{\prime} \mathbf{R}_{\mathbf{l}} \boldsymbol{\mu} / \lambda$ 
and $\mathbf{R}_{\mathbf{l}}=\boldsymbol{\Sigma}^{-1}-\boldsymbol{\Sigma}^{-1} \mathbf{l l}^{\prime} \boldsymbol{\Sigma}^{-1} / \mathbf{l}^{\prime} \boldsymbol{\Sigma}^{-1} \mathbf{l}$. The random variables $u_{1}, u_{2}$ and $u_{3}$ are independently distributed.

From Lemma 3.1 the distribution of $\mathbf{l}^{\prime} \mathbf{S}^{-1} \mathbf{x}$ is characterized in terms of a $\chi^{2}$ variable, a standard normal and a non-central $\mathcal{F}$ random variables which are independently distributed. As in the representation in Theorem 3.1, Section 3.1, the stochastic representation in Lemma 3.1 is computational faster as values for the product $\mathbf{l}^{\prime} \mathbf{S}^{-1} \mathbf{x}$ are generated from standard univariate distributions. The representation in Lemma 3.1 is useful in obtaining expressions for higher order moments of the product $\mathbf{l}^{\prime} \mathbf{S}^{-1} \mathbf{x}$. The result in the following Theorem 3.2 in Paper D is used to obtain formulas for the higher order noncentral and central moments of the estimated TP weights. Then we have the following theorem where higher order moments of $\mathbf{l}^{\prime} \mathbf{S}^{-1} \mathbf{X}$ are considered.

\section{Theorem 3.2 (Paper D)}

Let $\mathbf{S} \sim W_{p}(n, \boldsymbol{\Sigma}), n>p$ and $\mathbf{x} \sim N_{p}(\boldsymbol{\mu}, \lambda \boldsymbol{\Sigma})$ with $\lambda>0$ and positive definite $\boldsymbol{\Sigma}$. Furthermore, let $\mathbf{S}$ and $\mathbf{x}$ be independent and $\mathbf{l}$ be a $k$-dimensional vector of constants. Then the r-th order moment of $\mathbf{1}^{\prime} \mathbf{S}^{-1} \mathbf{x}$ is given by

$$
\begin{aligned}
\mathbb{E}\left[\left(\mathbf{l}^{\prime} \mathbf{S}^{-1} \mathbf{x}\right)^{r}\right] & =\frac{1}{(n-p-1) \ldots(n-p-2 r+1)}\left[\left(\mathbf{l}^{\prime} \boldsymbol{\Sigma}^{-1} \boldsymbol{\mu}\right)^{r}+\sum_{j=1}^{\lfloor r / 2\rfloor}\left(\begin{array}{c}
r \\
2 j
\end{array}\right) \frac{(2 j) !}{2^{j} j !}\right. \\
& \left.\times\left(\mathbf{l}^{\prime} \boldsymbol{\Sigma}^{-1} \boldsymbol{\mu}\right)^{r-2 j}\left(\lambda \mathbf{l}^{\prime} \boldsymbol{\Sigma}^{-1} \mathbf{l}\right)^{j}\left(1+\sum_{m=1}^{j}\left(\begin{array}{c}
j \\
m
\end{array}\right) c_{m}\right)\right]
\end{aligned}
$$

for $n-p+1>2 r$ with

$$
c_{m}=\frac{(p-1+2(m-1)) \ldots(p-1)}{(n-p-2(m-1)) \ldots(n-p)} e^{-\frac{s}{2}}{ }_{1} F_{1}\left(m+\frac{p-1}{2} ; \frac{p-1}{2} ; \frac{s}{2}\right),
$$

where $s=\boldsymbol{\mu}^{\prime} \mathbf{R}_{\mathbf{l}} \boldsymbol{\mu} / \lambda, \mathbf{R}_{\mathbf{l}}=\boldsymbol{\Sigma}^{-1}-\boldsymbol{\Sigma}^{-1} \mathbf{l l}^{\prime} \boldsymbol{\Sigma}^{-1} / \mathbf{l}^{\prime} \boldsymbol{\Sigma}^{-1} \mathbf{l}$ and ${ }_{1} F_{1}(\cdot ; \cdot ; \cdot)$ is the confluent hypergeometric function of the first order. 



\section{4}

\section{Concluding Remarks}

\subsection{Summary of contributions}

In this thesis linear discriminant analysis has been considered in large dimensions and especially with application to repeated measurements, e.g., growth curves. The goal has been focused on the development of new methods, such as a stochastic representation of the linear discriminant function coefficients and an approximation of misclassification errors.

The main contributions of the thesis are as follows:

- In Paper A, we derive a stochastic representation of the linear discriminant function coefficients which is based on random variables from standard univariate distributions. We apply the representation to approximate the error rate. We extend the results to large dimension asymptotics under the assumption that the dimension $p$ increases together with the sample size $n$ and they all tend to infinity, that is, $p / n$ converges to a positive constant $c \in(0,1)$.

- In Paper B, we use the linear discriminant function with the mean following the Growth Curve model to derive results for the approximation of the misclassification errors. We investigate the performance of the derived approximations through a Monte Carlo study. When $\boldsymbol{\Sigma}$ is known we note a better classification with an increase in repeated measurements. For an unknown $\Sigma$, a better classification with fewer repeated measurements is noted.

- In Paper C, we propose a linear classification rule for the Growth Curve model with restrictions on the mean parameter space. We extend results of Paper B by deriving 
an approximation of the misclassification errors under the Extended Growth Curve model. Furthermore, we derive a classification rule under rank restriction on the mean parameter matrix.

- A function of a Wishart matrix and a normal vector applied in Paper A (discriminant function coefficients) is considered in paper $\mathrm{D}$, where its applications appears in portfolio theory. We derive analytical expressions for higher order central moments and non-central moments of the portfolio weights. We provide analytical results for higher order moments of the estimated tangency portfolio weights.

\subsection{Future research}

There are several potential research problems related to this thesis.

In Paper A we study the distributional properties of the linear discriminant function under a normality assumption where two groups with the same covariance matrix but different mean vectors are compared. This paper can be extended by deriving theoretical results to the case of a quadratic discriminant function where two groups have different covariance matrices. This approach will require the development of new stochastic representations.

The linear discriminant function used in Paper B can be extended to linear discriminant function with the group means following the random effects Growth Curve model. Both in Paper B and C we have used known covariance matrices. Of course it is of great interest to study the case of an unknown covariance matrix for the high dimensional case employing the Moore-Penrose generalized inverse or regularizations approaches such as a ridge-type based approach.

In Paper D we have considered financial stocks (features) when they are less than the number of observations in the portfolio. But the problem can be extended to consider a number of financial stocks comparable to the number of observations in the portfolio. This problem will mostly use large dimension asymptotic approaches .

Finally, application of the developed results in this thesis to real life data sets and a comparison with the existing ones may be paramount to show their merits. 


\section{Bibliography}

Aggarwal, C. C. (2018). Neural Networks and Deep Learning: A Textbook. Springer, Yorktown Heights, New York.

Anderson, T. W. (1951). Classification by multivariate analysis. Psychometrika, 16(1):31-50.

Anderson, T. W. (2003). An Introduction to Multivariate Statistical Analysis, Third edition. Wiley Series in Probability and Statistics, New York.

Bodnar, T. and Okhrin, Y. (2011). On the product of inverse Wishart and normal distributions with applications to discriminant analysis and portfolio theory. Scandinavian Journal of Statistics, 38(2):311-331.

Bodnar, T. and Schmid, W. (2009). Estimation of optimal portfolio compositions for Gaussian returns. Statistics \& Decisions International Mathematical Journal for Stochastic Methods and Models, 26(3):179-201.

Britten-Jones, M. (1999). The sampling error in estimates of mean-variance efficient portfolio weights. The Journal of Finance, 54(2):655-671.

Bun, J., Bouchaud, J.-P., and Potters, M. (2017). Cleaning large correlation matrices: tools from random matrix theory. Physics Reports, 666:1-109.

Burnaby, T. (1966). Growth-invariant discriminant functions and generalized distances. Biometrics, 22:96-110. 
Deev, A. (1970). Representation of statistics of discriminant analysis, and asymptotic expansion when space dimensions are comparable with sample size. Doklady Akademii Nauk, 195(4):759-762.

Efron, B. (2006). Minimum volume confidence regions for a multivariate normal mean vector. Journal of the Royal Statistical Society: Series B, 68(4):655-670.

Fama, E. and French, K. (2012). Questions and Answers: Are stock returns normally distributed? Fama/French Forum. http:famafrench.dimensional.com/questionsanswers/qa-are- stock-returns-normally-distributed.aspx. Accessed 2019-12-27.

Fama, E. F. (1976). Efficient Capital Markets-Portfolio Decisions and Securities Prices. Basic Books, New York.

Fan, J., Liao, Y., and Liu, H. (2016). An overview of the estimation of large covariance and precision matrices. The Econometrics Journal, 19(1):1-32.

Filipiak, K. and von Rosen, D. (2012). On maximum likelihood estimators in an extended multivariate linear Growth Curve model. Metrika, 75(8):1069-1092.

Fisher, R. A. (1936). The use of multiple measurements in taxonomic problems. Annals of Eugenics, 7:179-188.

Fisher, R. A. (1938). The statistical utilization of multiple measurements. Annals of Eugenics, 8(4):376-386.

Friedman, J. H. (1989). Regularized discriminant analysis. Journal of the American Statistical Association, 84:165-175.

Fujikoshi, Y. (2000). Error bounds for asymptotic approximations of the linear discriminant function when the sample sizes and dimensionality are large. Journal of Multivariate Analysis, 73:1-17.

Fujikoshi, Y. and Seo, T. (1998). Asymptotic aproximations for EPMC of the linear and the quadratic discriminant functions when the sample sizes and the dimension are large. Random Operators and Stochastic Equations, 6(3):269-280.

Fujikoshi, Y., Ulyanov, V. V., and Shimizu, R. (2010). Multivariate Statistics: HighDimensional and Large-Sample Approximations. Wiley, New York.

Ghatak, A. (2019). Deep Learning with R. Springer, Kolkata.

Gupta, A. and Nagar, D. (2000). Matrix Variate Distributions. Chapman and Hall/CRC, Boca Raton. 
Gupta, S. D. (1968). Some aspects of discrimination function coefficients. Sankhyā: The Indian Journal of Statistics, Series A, 30:387-400.

Gutierrez, D. D. (2015). Machine Learning and Data Science: An Introduction to Statistical Learning Methods with R. Technics Publications, Basking Ridge, New Jersey.

Haff, L. (1982). Identities for the inverse Wishart distribution with computational results in linear and quadratic discrimination. Sankhyā: The Indian Journal of Statistics, Series B, 44:245-258.

Hastie, T., Tibshirani, R., and Friedman, J. (2009). The Elements of Statistical Learning: Data Mining, Inference, and Prediction. Springer Science \& Business Media, Stanford, California.

Hotelling, H. (1931). The generalization of students ratio. Annals of Mathematical Statatistics, 2:360-378.

Hyodo, M., Mitani, T., Himeno, T., and Seo, T. (2015). Approximate interval estimation for EPMC for improved linear discriminant rule under high dimensional frame work. SUT Journal of Mathematics, 51(2):145-179.

Ingersoll, J. E. (1987). Theory of Financial Decision Making. Rowman \& Littlefield Publishers.

James, G., Witten, D., Hastie, T., and Tibshirani, R. (2013). An Introduction to Statistical Learning: with Applications in R. Springer, New York.

John, S. (1961). Errors in discrimination. The Annals of Mathematical Statistics, 32:1125-1144.

Johnson, R. A. and Wichern, D. W. (2007). Applied Multivariate Statistical Analysis. Prentice Hall Upper Saddle River, New Jersey.

Khatri, C. G. (1966). A note on a MANOVA model applied to problems in growth curve. Annals of the Institute of Statistical Mathematics, 18(1):75-86.

Kollo, T. and von Rosen, D. (2005). Advanced Multivariate Statistics with Matrices, volume 579. Springer Science, New York.

Kotsiuba, I. and Mazur, S. (2016). On the asymptotic and approximate distributions of the product of an inverse Wishart matrix and a Gaussian vector. Theory of Probability and Mathematical Statistics, 93:103-112. 
Lachenbruch, P. A. (1968). On expected probabilities of misclassification in discriminant analysis, necessary sample size, and a relation with the multiple correlation coefficient. Biometrics, 24:823-834.

Lee, J. C. (1977). Bayesian classification of data from growth curves. South African Statistical Journal, 11(2):155-166.

Lee, J. C. (1982). Classification of growth curves. Handbook of Statistics, Vol. II. P. R. Krishnaiah and L. W. Kanal (Eds)., volume 2. North-Holland.

Mahalanobis, P. C. (1936). On the generalized distance in statistics. National Institute of Science of India, 2:49-55.

Markowitz, H. (1952). Portfolio selection. Journal of Finance, 7:77-91.

McLachlan, G. (1977). Constrained sample discrimination with the studentized classification statistic. Communications in Statistics-Theory and Methods, 6(6):575-583.

Mentz, G. B. and Kshirsagar, A. M. (2005). Classification using growth curves. Communications in Statistics-Theory and Methods, 33(10):2487-2502.

Muirhead, R. J. (1982). Aspects of Multivariate Statistical Theory. Wiley, New York.

Nagel, D. (1979). Bayesian classification estimation and prediction of growth curves. South African Statistical Journal, 13(2):127-137.

Okamoto, M. (1963). An asymptotic expansion for the distribution of the linear discriminant function. The Annals of Mathematical Statistics, 34(4):1286-1301.

Pavlenko, T. and von Rosen, D. (2001). Effect of dimensionality on discrimination. Statistics, 35(3):191-213.

Pearson, K. (1916). On the probability that two independent distributions of frequency are really samples from the same population. Biometrika, 8(1):250-254.

Potthoff, R. F. and Roy, S. (1964). A generalized multivariate analysis of variance model useful especially for growth curve problems. Biometrika, 51:313-326.

Rao, C. R. (1967). Least squares theory using an estimated dispersion matrix and its application to measurement of signals. Proceedings of the fifth Berkeley symposium on mathematical statistics and probability, 1:355-372.

Rao, C. R. (1970). Inference on discriminant function coefficients. Essays in Probability and Statistics, 30:587-602. 
Raudys, Š. (1967). On determining the training sample size of a linear classifier. Computing systems, 28:79-87.

Raudys, Š. (1972). On the amount of a priori information in designing the classification algorithm. Proc. Academy of Sciences of the USSR: Engineering Cybernetics, 4:168174.

Raudys, Š. (2012). Statistical and Neural Classifiers: An Integrated Approach to Design. Springer Science \& Business Media.

Raudys, Š. and Jain, A. (1991). Small sample size effects in statistical pattern recognition: Recommendations for practitioners. IEEE Transactions on Pattern Analysis \& Machine Intelligence, 3:252-264.

Raudys, Š. and Young, D. M. (2004). Results in statistical discriminant analysis: A review of the former soviet union literature. Journal of Multivariate Analysis, 89(1):1-35.

Rencher, A. C. and Christensen, W. (2012). Methods of Multivariate Analysis. Wiley, Toronto, Canada.

Roy, A. and Khattree, R. (2005a). Discrimination and classification with repeated measures data under different covariance structures. Communications in StatisticsSimulation and Computation, 34(1):167-178.

Roy, A. and Khattree, R. (2005b). On discrimination and classification with multivariate repeated measures data. Journal of Statistical Planning and Inference, 134(2):462-485.

Roy, A. and Khattree, R. (2007). Classification of multivariate repeated measures data with temporal autocorrelation. Journal of Applied Statistical Science, 15(3):283-294.

Shutoh, N., Hyodo, M., and Seo, T. (2011). An asymptotic approximation for epmc in linear discriminant analysis based on two-step monotone missing samples. Journal of Multivariate Analysis, 102(2):252-263.

Tu, J. and Zhou, G. (2004). Data-generating process uncertainty: What difference does it make in portfolio decisions? Journal of Financial Economics, 72(2):385-421.

Verbyla, A. P. and Venables, W. (1988). An extension of the Growth Curve model. Biometrika, 75(1):129-138.

von Rosen, D. (1988). Moments for the inverted Wishart distribution. Scandinavian Journal of Statistics, (15):109-109.

von Rosen, D. (1989). Maximum likelihood estimators in multivariate linear normal models. Journal of Multivariate Analysis, 31(2):187-200. 
von Rosen, D. (2018). Bilinear Regression Analysis: An Introduction. Springer, New York.

Wald, A. (1944). On a statistical problem arising in the classification of an individual into one of two groups. The Annals of Mathematical Statistics, 15:145-162.

Watanabe, H., Hyodo, M., Seo, T., and Pavlenko, T. (2015). Asymptotic properties of the misclassification rates for euclidean distance discriminant rule in high-dimensional data. Journal of Multivariate Analysis, 140:234-244.

Wyman, F. J., Young, D. M., and Turner, D. W. (1990). A comparison of asymptotic error rate expansions for the sample linear discriminant function. Pattern Recognition, 23:775-783. 
Part II

\section{Papers}




\section{Papers}

The papers associated with this thesis have been removed for copyright reasons. For more details about these see:

http://urn.kb.se/resolve?urn=urn:nbn:se:liu:diva-165558 
